


$\left|\begin{array}{l|l|l|}2500 \\ 2600 \\ 2700 \\ 2800 \\ 2900 \\ 3000 \\ 3100 \\ 3200 \\ 3300 \\ 3500 \\ 3500 \\ 3500 \\ 3500\end{array}\right|$

SERIAL TEMPERATURES.

\begin{tabular}{|c|c|c|c|c|}
\hline ВРTH. & TEMP. & NO. OF THER. & COR. & COR. TEMP. \\
\hline 25 & & & & \\
\hline 50 & & & & \\
\hline 100 & & & & \\
\hline 200 & & & & \\
\hline 300 & & & & \\
\hline 400 & & & & \\
\hline 500 & & & & \\
\hline 600 & & & & . \\
\hline 700 & & & & \\
\hline 800 & & & & \\
\hline 900 & & & & \\
\hline 1000 & & & & \\
\hline
\end{tabular}

REMARKS : 


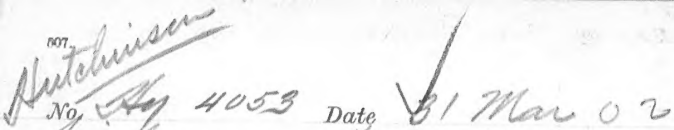
together Machine. \#, Reel. How ry Turns 94 corr of Depth 99 fuss

stow lead $36 \mathrm{lg}$.

Bottom for $\mathcal{L}$. Sh

Bottom temperature 68.50

No. of thermometer 92442 Cor.

Corrected temperature

Air 79 surface $\% 5$ Drift

Trawl or dredge

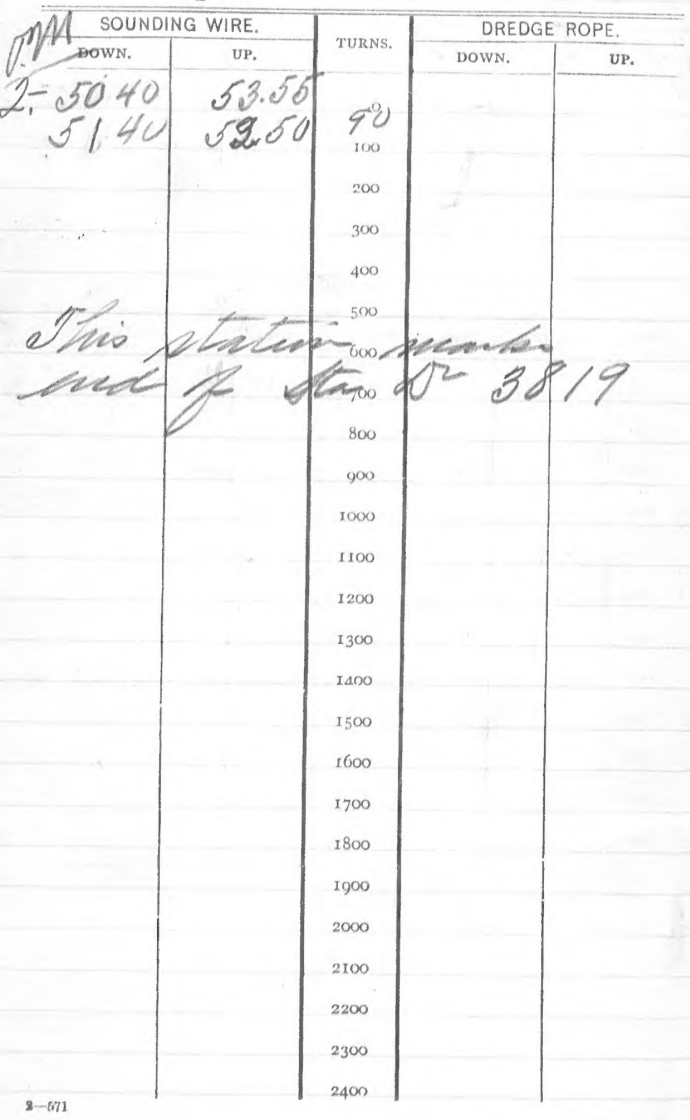




$$
\frac{1}{15}
$$


Whortumise Dat fig. 3.4

No. 503820 Date 31 Mare 02 Eugefer Machine." / Reel. Whammy Turns 59 cor. 6 Depth 65 fun s stroton lead 36 lino. Bottom free, e. S, hl - Sh. Bottom temperature $72^{\circ}$ No. of thermometer 92442 Cor. Corrected temperature

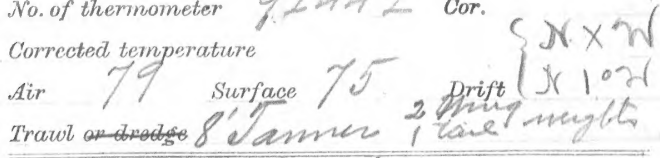

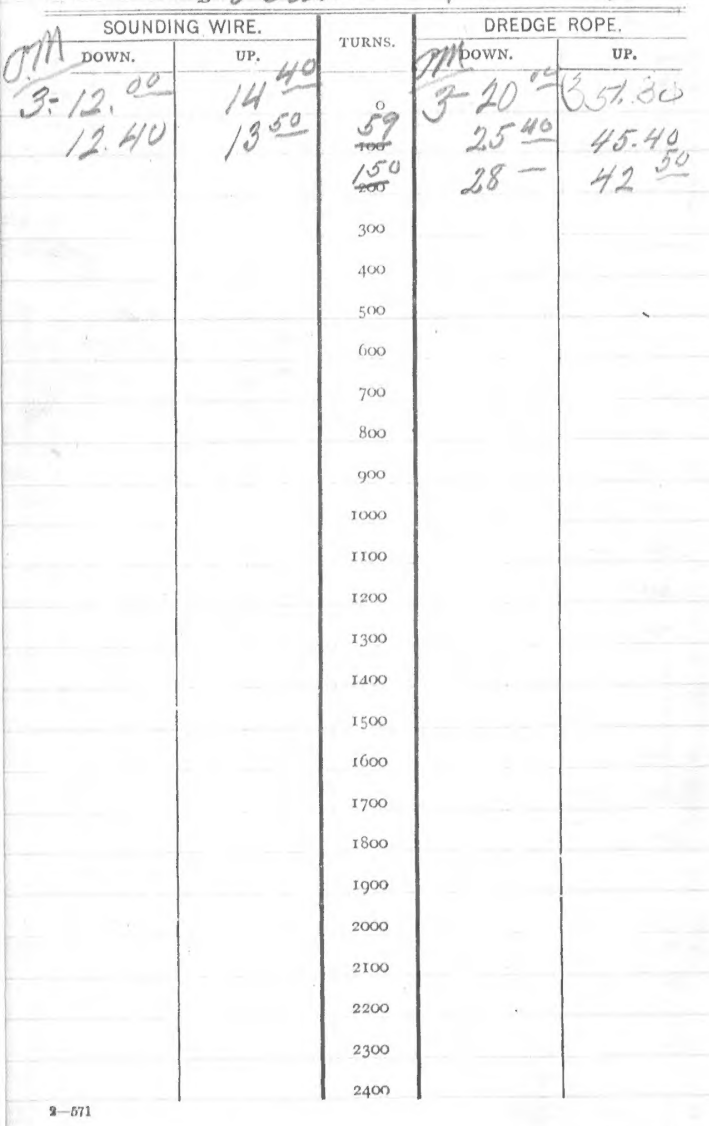




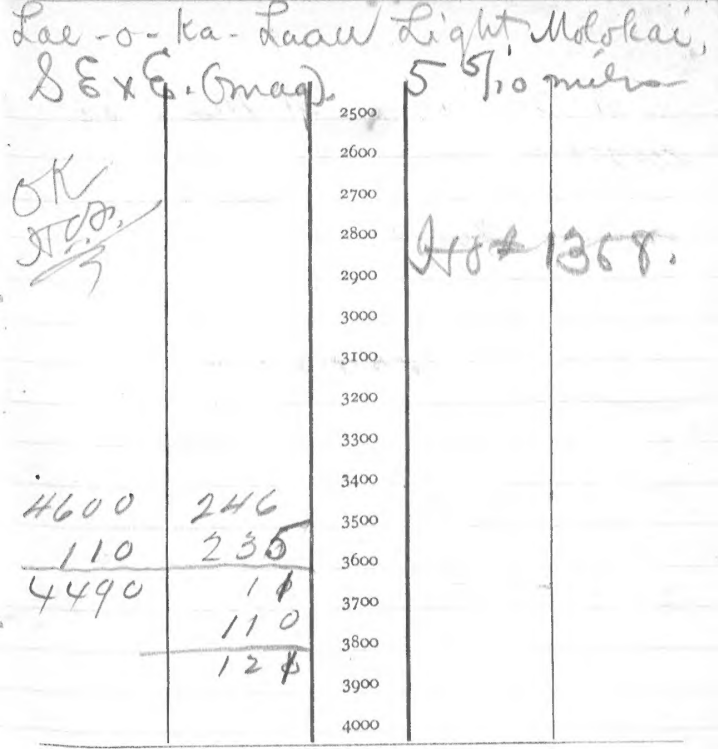

SERIAL TEMPERATURES.

\begin{tabular}{|c|c|c|c|c|}
\hline DEPTH. & TEMP. & NO, OF THER. & COR. & COR. TEMP. \\
\hline 25 & & & & \\
\hline 50 & & & & \\
\hline 100 & & & & \\
\hline 200 & & & & \\
\hline 300 & & & & \\
\hline 400 & & & & \\
\hline 500 & & & & \\
\hline 600 & & & & \\
\hline 700 & & & & \\
\hline 800 & & & & \\
\hline 900 & & & & \\
\hline 1000 & & & & \\
\hline
\end{tabular}

REMARKS : 
stover

... $D^{2}-3821$.

Date 31 Hel 02.

Machine.

Turns Cor. +
Reel.

Depth 6 fino -

Bottom ers.

Bottom temperature

No. of thermometer

Corrected temperature

Air $76^{\circ}$ Surface $76^{\circ}$ Drift Whine

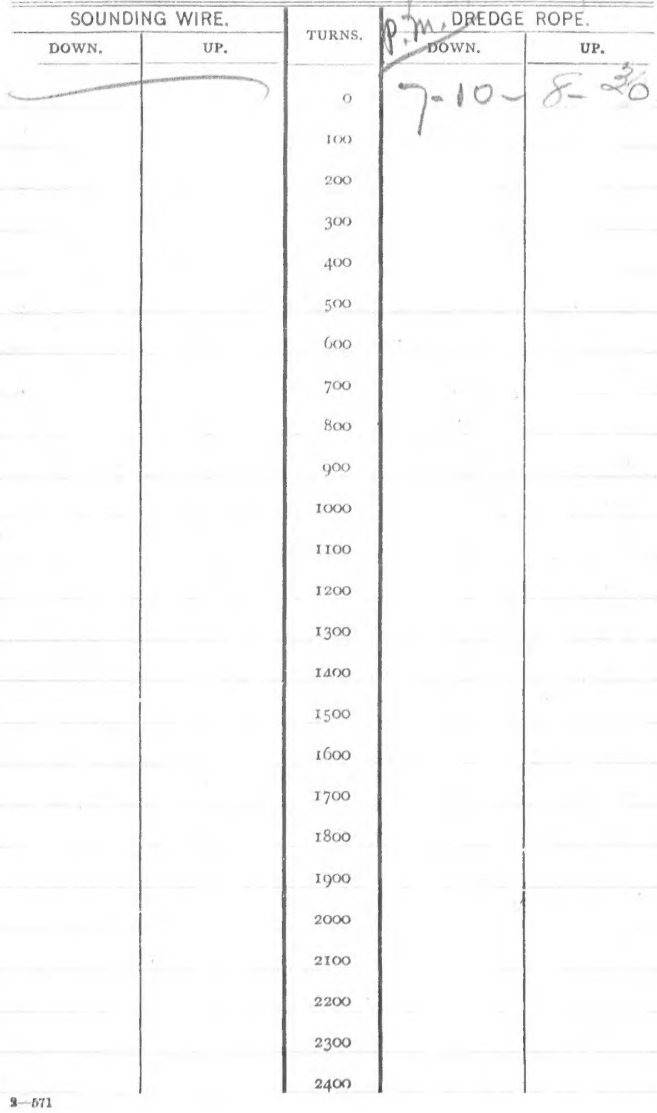


Anchorag, fouth const Molokai'Is.

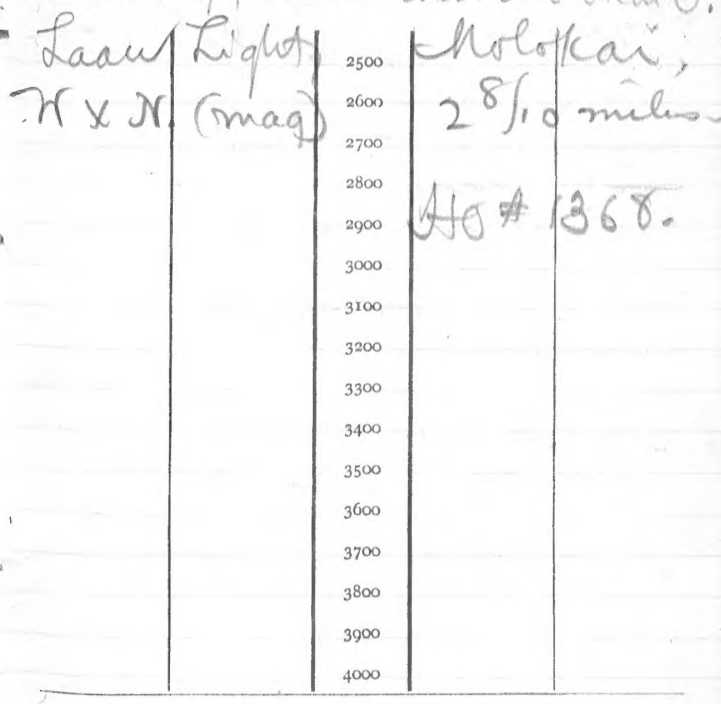

SERIAL TEMPERATURES,

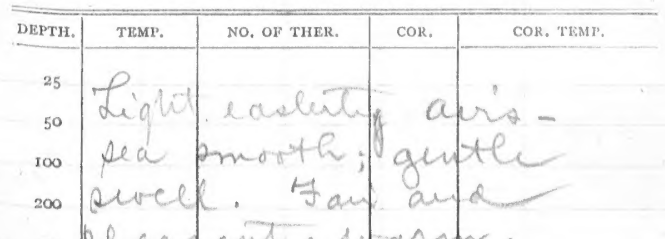

300 pecapant, Marpor.

${ }_{500}{ }^{00}$ Dip-net catch: defuonepis very 6oo abundafut; a few ther annclids many

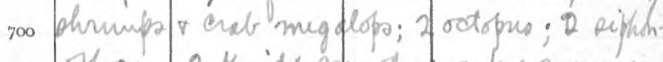
soo othorio: 2 kinids oynodud, a fur myxus, 9001 atherinta, 2 upreup (?) ; pany gobiorids; rooo peit blentroids- Lafqu ede Ablew dout REMARKS :

Also at Gobtu. pot in paud fration near ahip, from 630 . on to 630 an. oalthering brit - not disturbed - no catchFrom $6 t_{0} 7$ pow 7 haud hinis, salt herving bait, over side- no eatch. 
î $\mathrm{No}_{0}=4 \%$

Date

0.2

S ifsbee Machino Mo/Reel. Waw,
Turns 2l. Cor. +
2
Depth
23 for

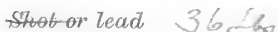

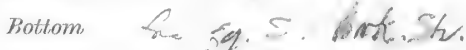

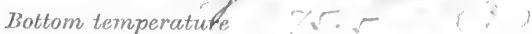

No. of thernometer 2, Cor.

Corrected ternperature
Air
Surface
Drift

Irawl or dredge

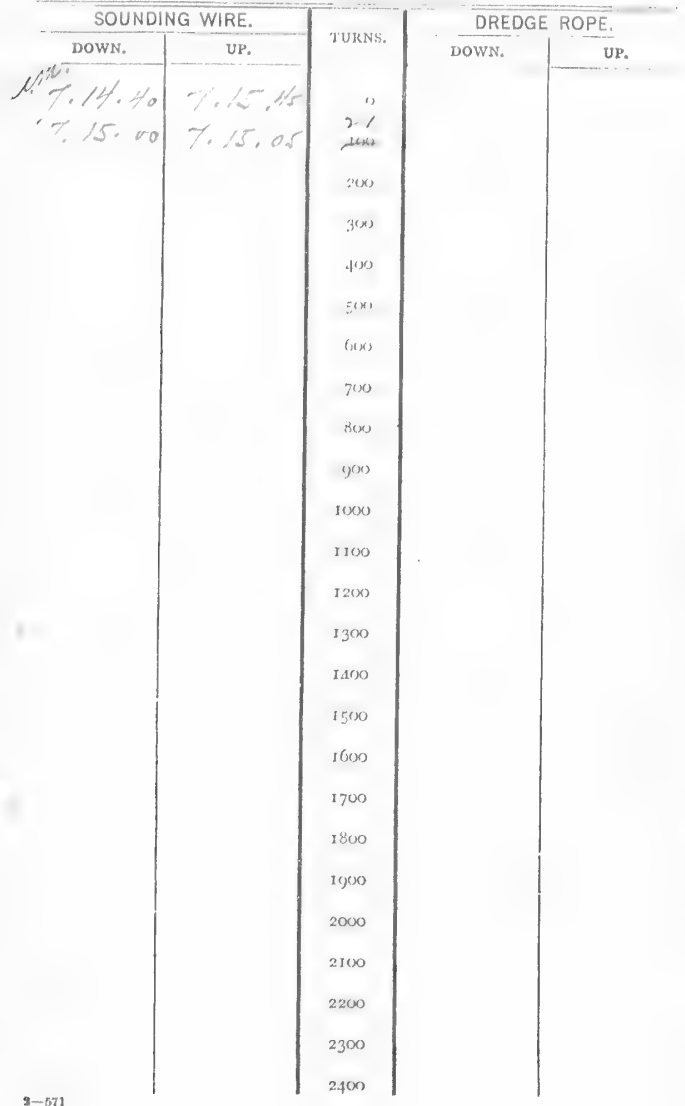




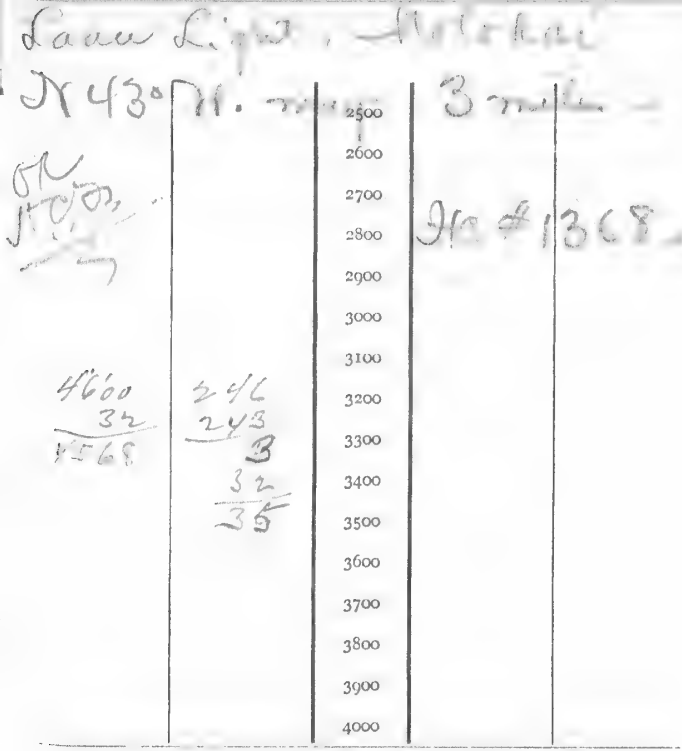

SERIAL TEMPERATURES.

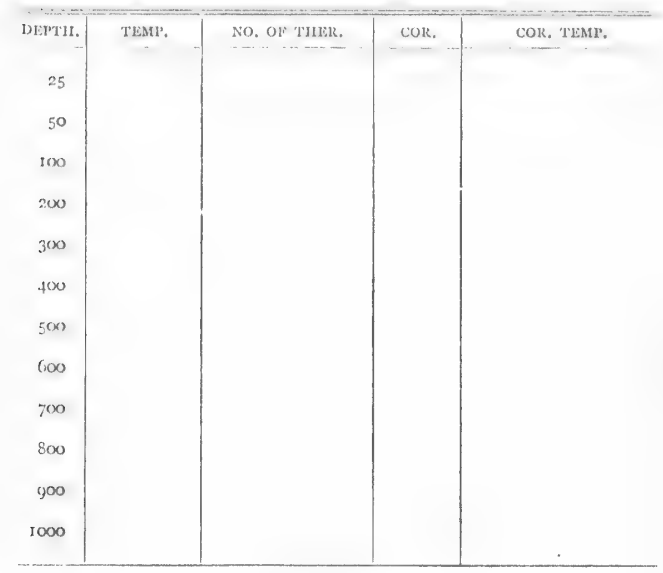

REMARKS : 
ror. . in inter

No. L2 2 2Date

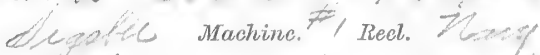

Therns $4 /$ Cor. +4 Dopth में 5 ,

Shoubar lead

Bottom

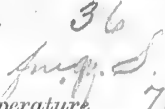

Bottom temperature

No. of thormometer. $\Rightarrow, i+i$ Cor.

\section{Corrected temperature}

Air Y? Surface

) 3,4 की

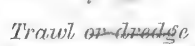

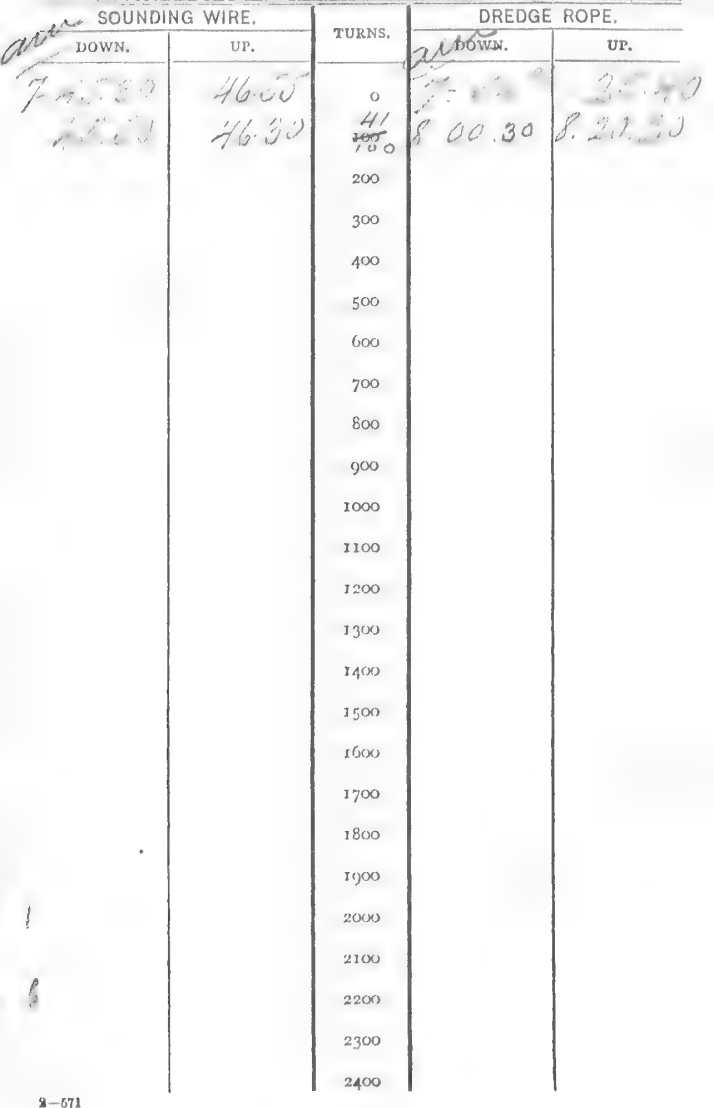




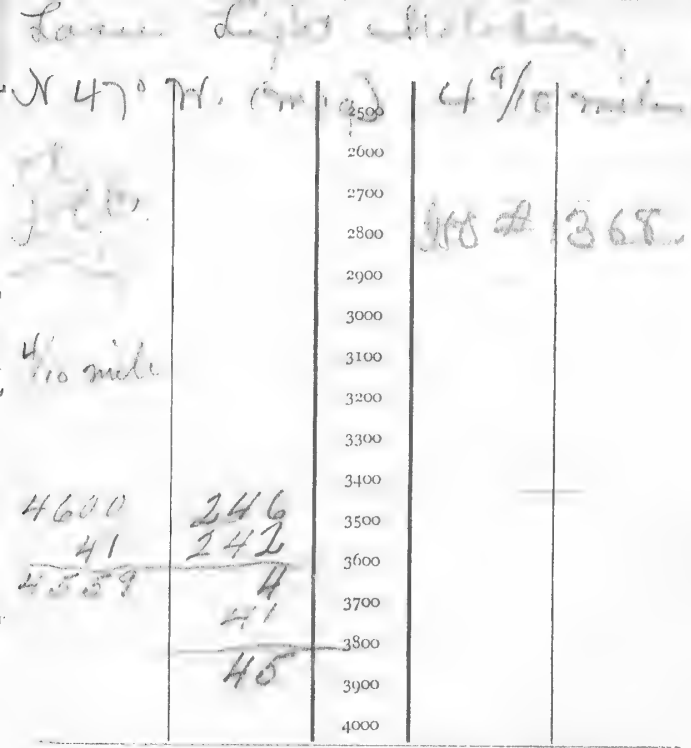

SERIAI. TLMPERATURES.

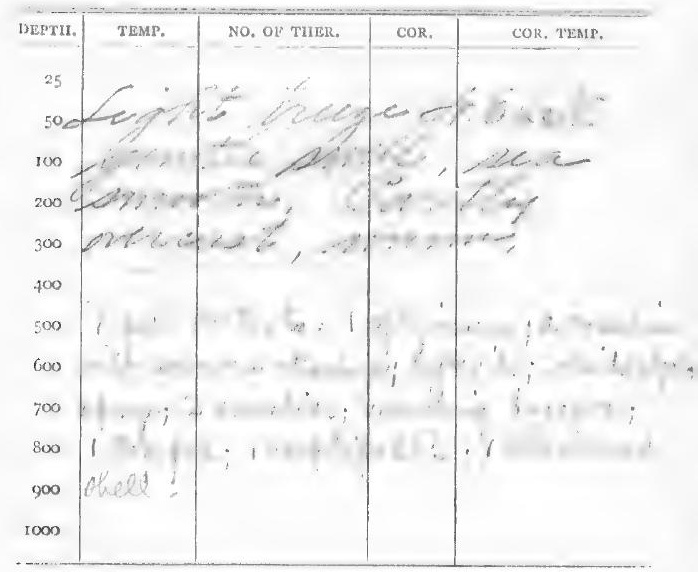

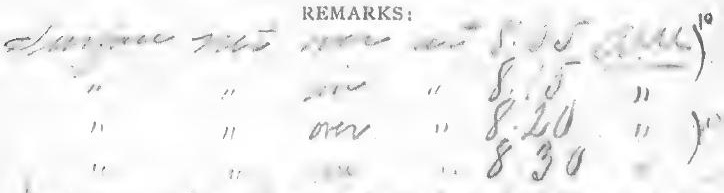
$|x|=$ r.

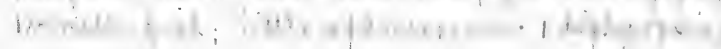
megaloto. 


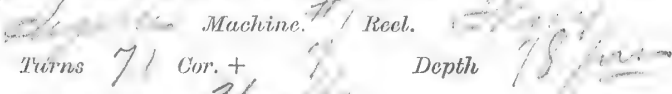

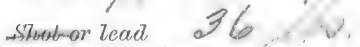

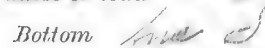

Bottom temperature $69^{\circ}$

No.of thermometer $\mathrm{C} 2 \mathrm{C} 2 \mathrm{cor}$.

Corrected tempercetsure

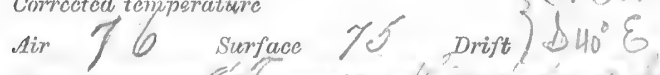

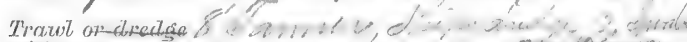

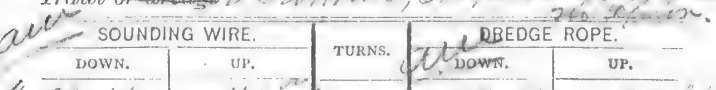

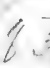

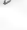

$$
\text { s. }
$$

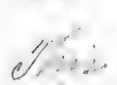

is

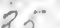

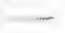

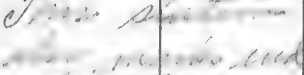

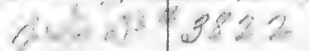

I

(n)

s.

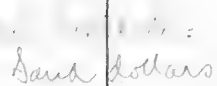
myeidiunt; 1 fish dicenar contenta

ij Getopins.

$1:$

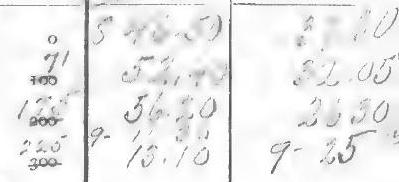

700

800

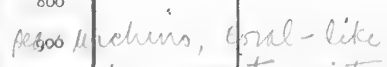

-

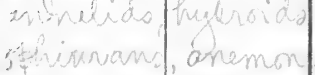
¿óleds, la taubifram

1200

1300

1400

1500

$\therefore 2000$ istant

I 700

1800

1), I000

12000

2100

2200

2300

2400 
darwaig dririca'

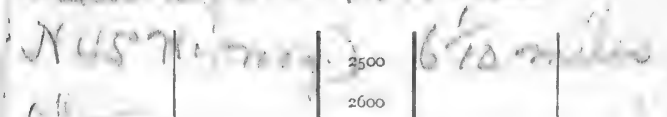

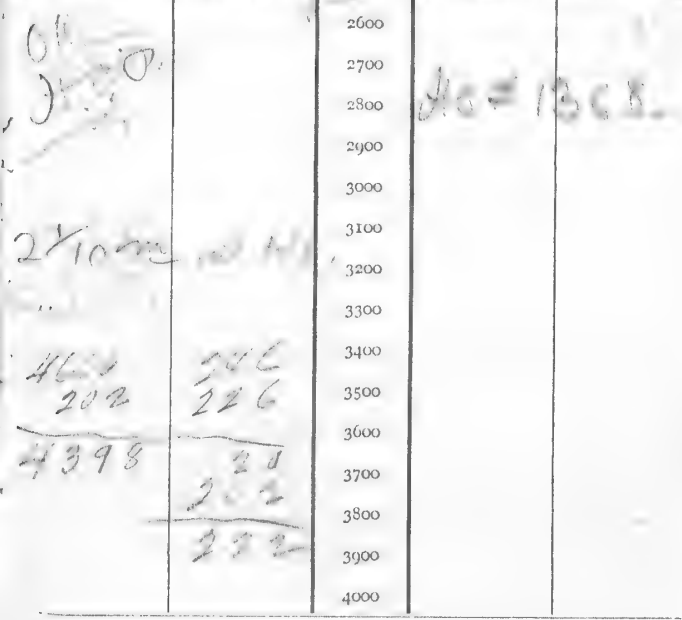

SERIAL TEMPERATURES.

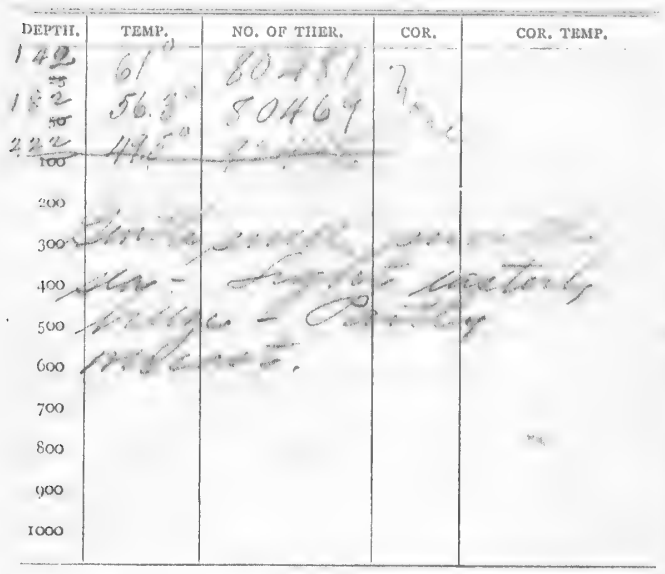

EAR , REMARKS:

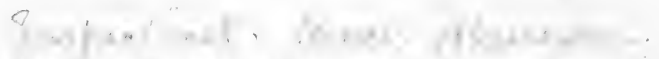

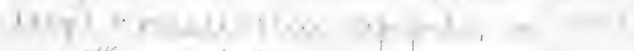

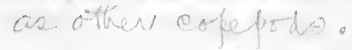




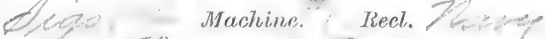

Turns 653 Cor. +40 Depth 498 his.

Statorat 86 is.

\section{Bottom}

Bottom temperature 62

Non of thormometer of. 2 it 2 Cor.

Corrected temperature

Air

cor.

tranus or dructgen

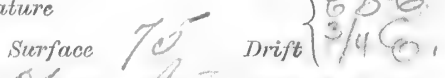

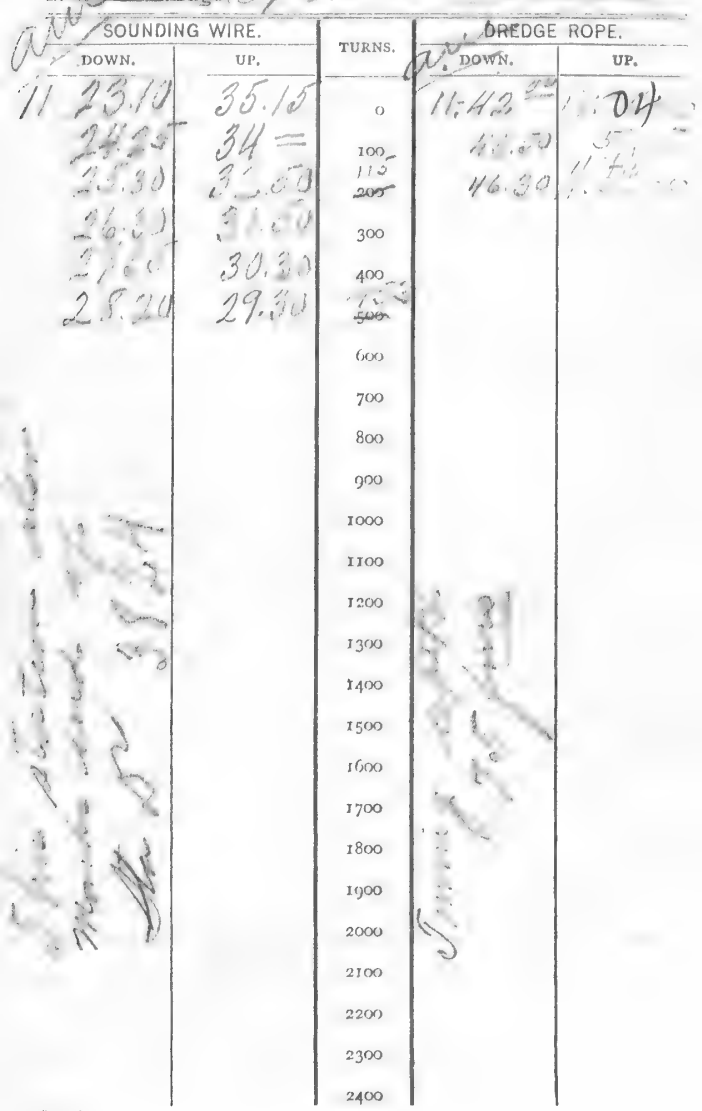




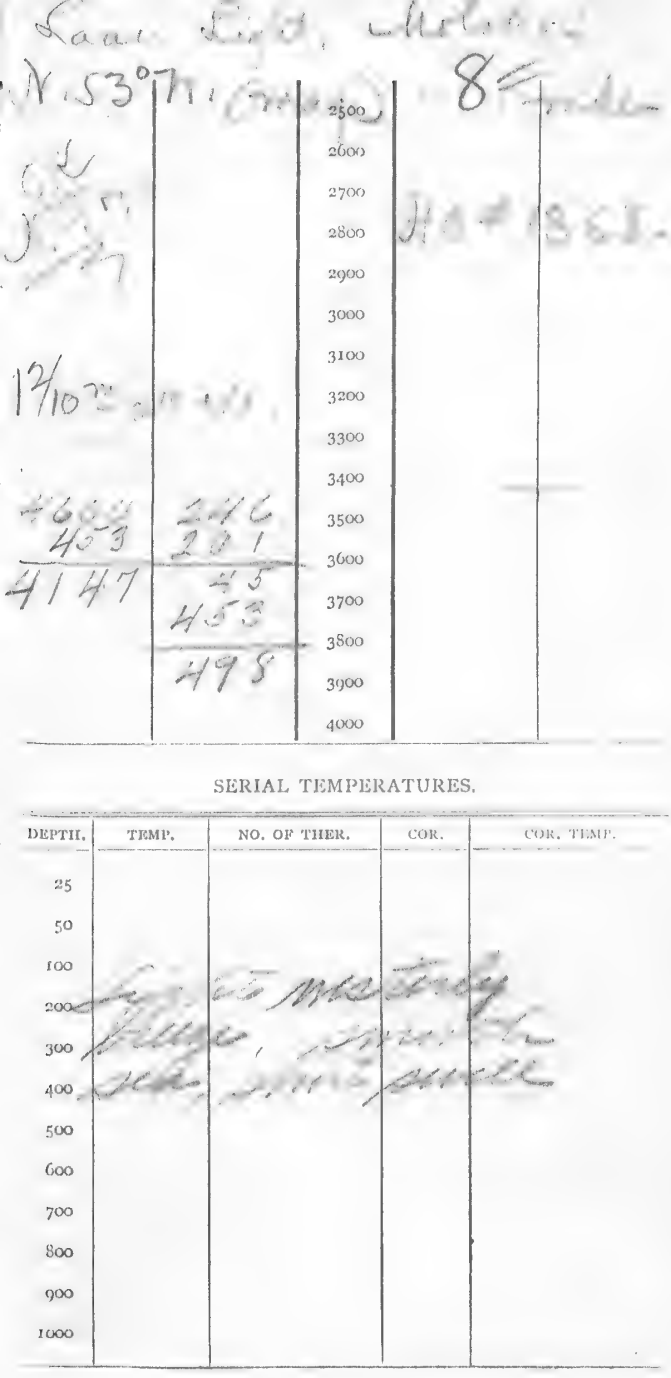

REMARKS :

1 "Hlounder ; 1 iuthrotoms; a - 


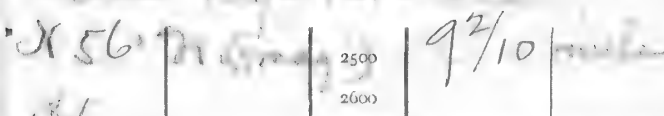
14. 2700 2000 3000 3100 3200 3300

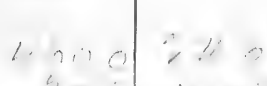

3.400

3500 3000 $\frac{35}{420}$ \begin{tabular}{ccc|c}
3 & 1 & 3700 \\
1 & 3 & 1 & 3800 \\
40000 & 1
\end{tabular} \mid

SERIAL TEMPLRATURES.

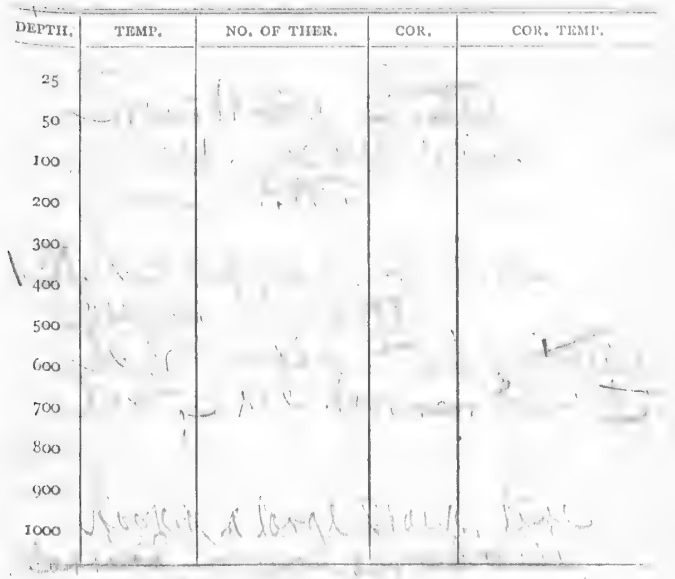

REMARKS :

.$$
\text { s }
$$

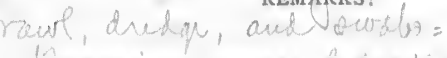

, His

1

10 grogonian. 



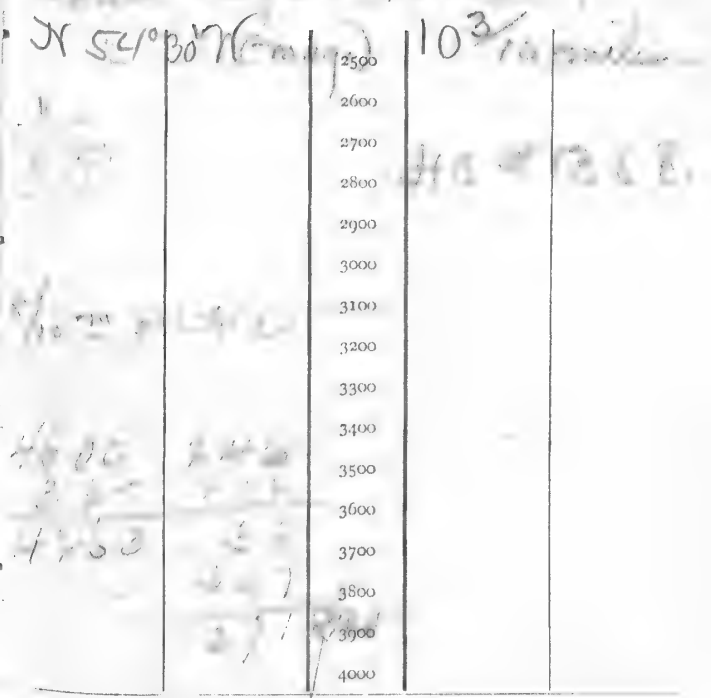

SERIAI. TEMPERATURJES.

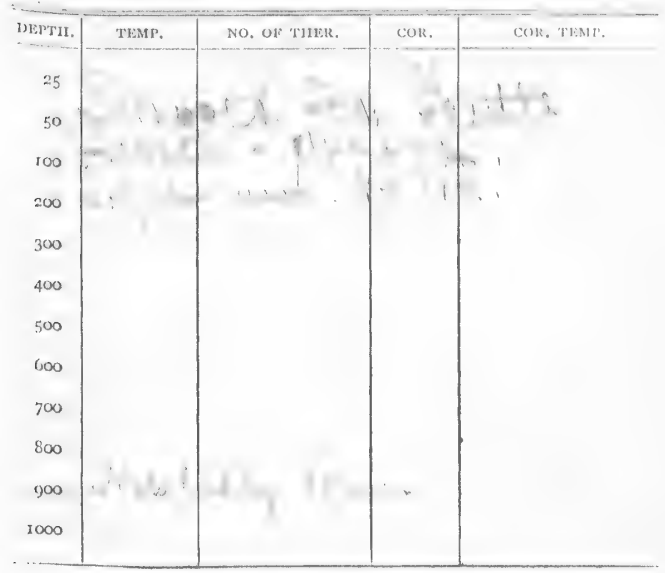

REMARKS :

- Oratle trank 1. ibo duedad:

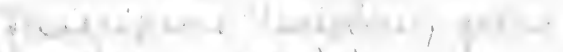





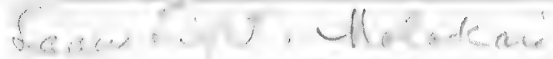

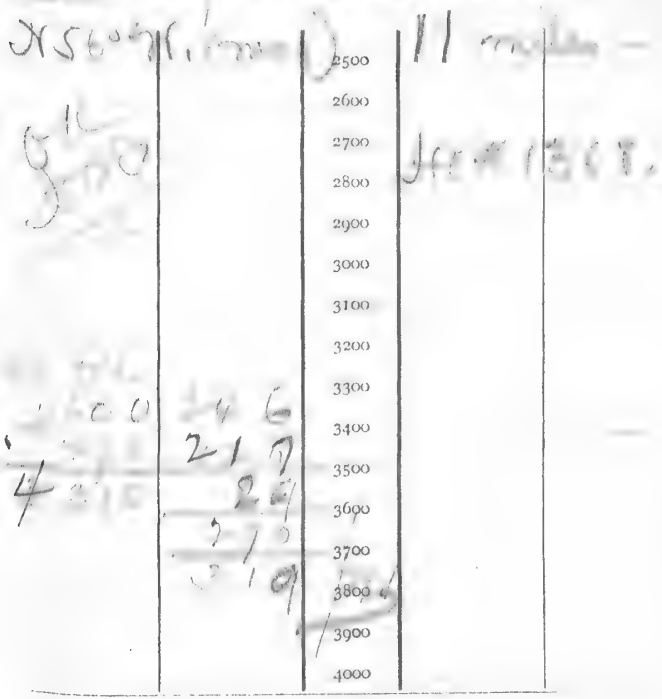

SERIAL, TEMPERATURES.

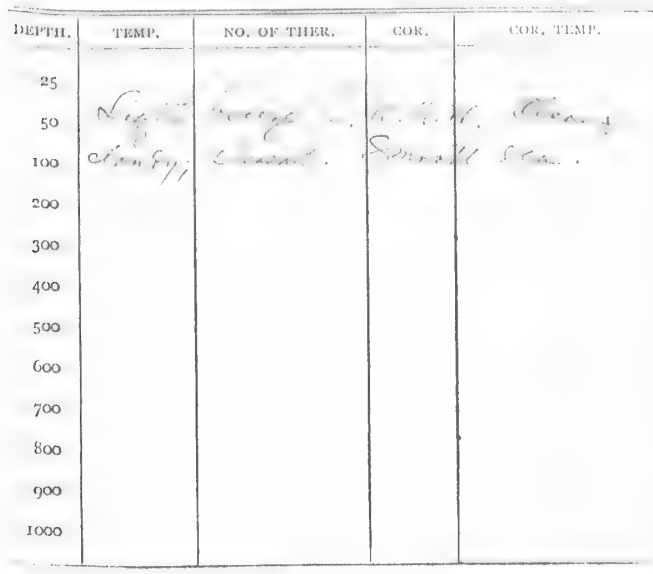

RFMARKS :

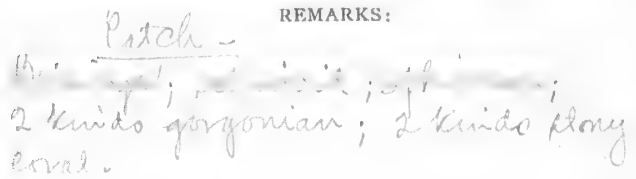


not. $\mid+i$

hro.

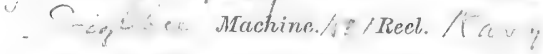
Therms 255 Cor. +
Depth 2-8!

Alsobor lead 3 lo 16 .

Bottom

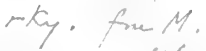

Boltom temperature

No of thermotneter

80481

Cor.

Corrected temperature
Air
Sterfuen
Drift

Trawt or dredge

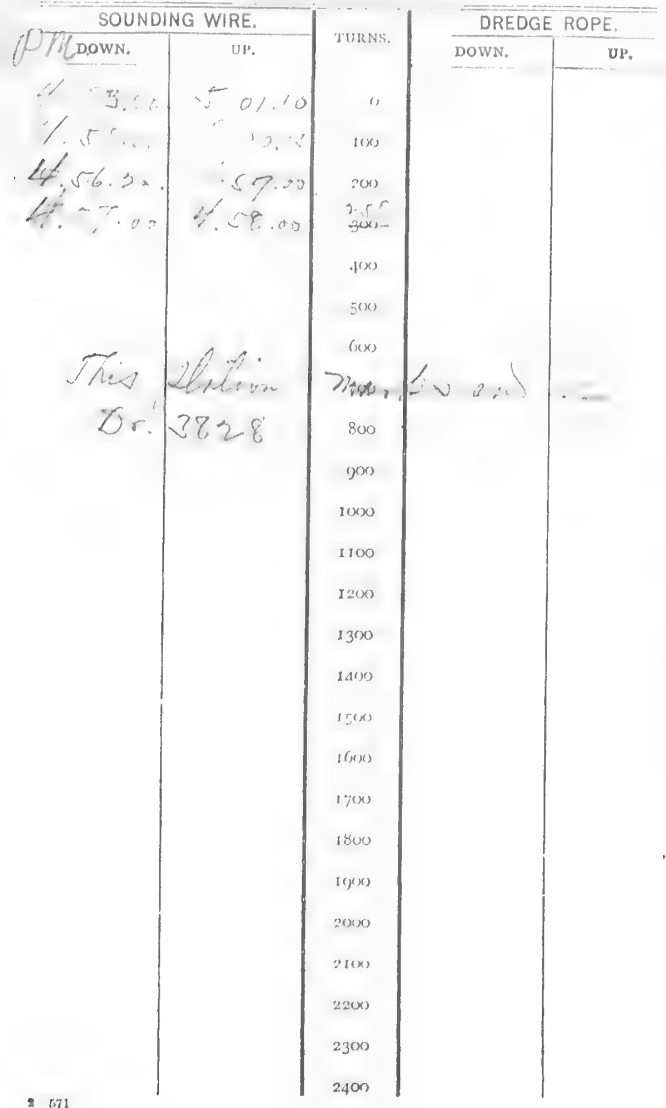




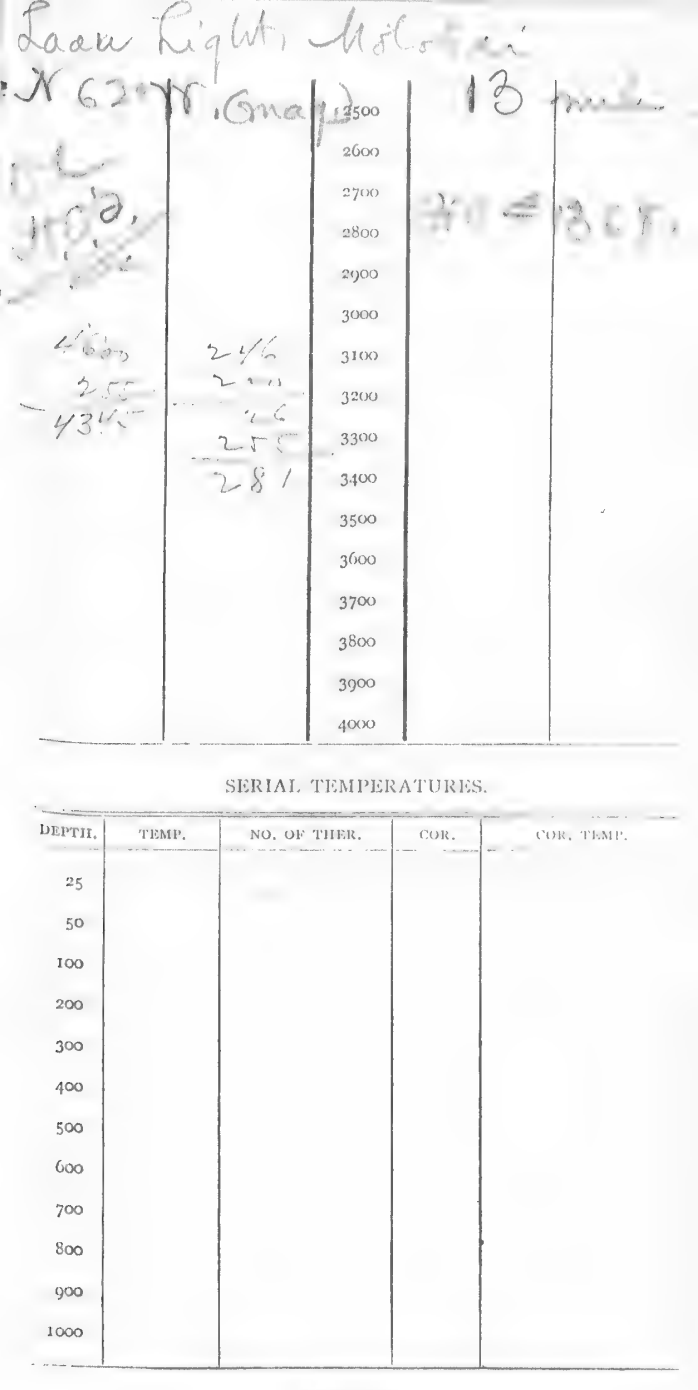


No. A , $38 \%$ i Date.

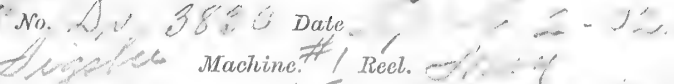
Turns 377 Cor. +25 Depth $\therefore 7$. storator lead

Bottom lot $/ \mathrm{m} \cdot \mathrm{ln}$

Bottom temperature 45,2

No. of thormometer 80481

Corrected temperature

Air Sierfaco

Hrower of dracterem

is

Cor.

$\ell 1^{\circ} \mathrm{C}$.

$5 \times 6$

SOUNDING WIRE.

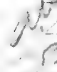

מOWN.

$2, \cdots \cdots+C, 16$

S

$4 \% 00 \% 0$

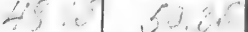

$29 .-20$

c50.11)

TURNS. \begin{tabular}{|l|l|l|l|l} 
DOWEDGE ROPE. \\
\hline DOWN.
\end{tabular}

- $\div 56,30 \quad 30$

J00

$2-0 / 20$

37.40

$0.5=$

33.5

200$$
\text { }
$$

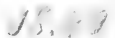

il 210

400

12.10

$9 .-7.2$

500

600

700

800

900

1000

I 100

I 200

1300

1400

1500

1000

1700

1800

I 900

2000

2100

2200

2300

2400 



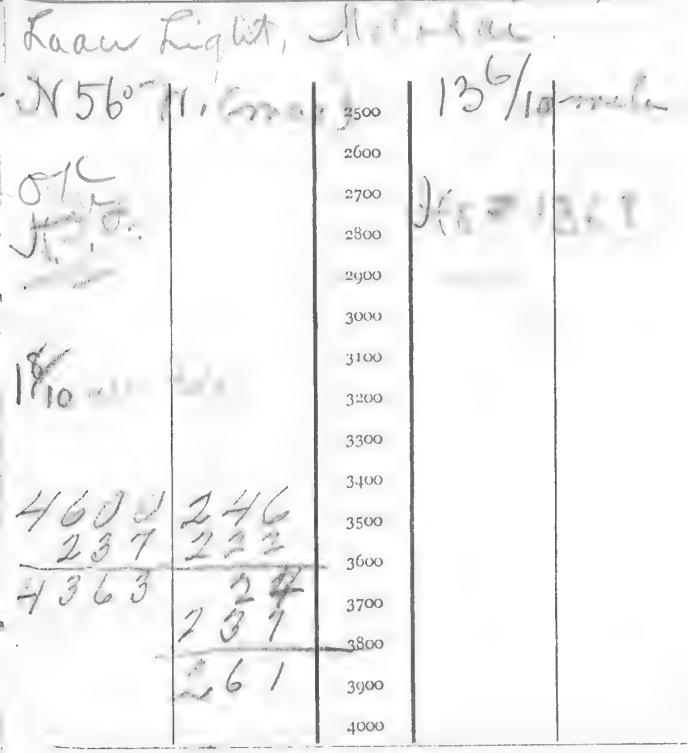

SERIAI, TEMIPERATURES.

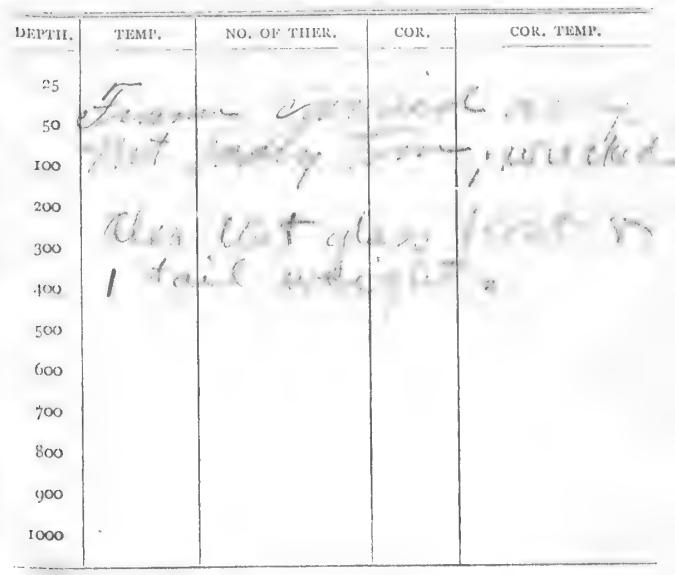

REMARKS :

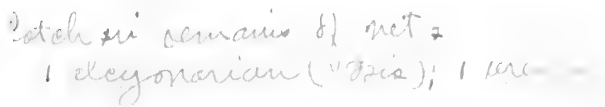


_... Machine f Turns 12 cor +16 Depth 178 (nwo

Stuab-or lead

Botiom letiri iplo.

Bottom temperature

No. of thormometer \& 481

Cor.

Corrected temperature

$\operatorname{Air} 74$ Surface 76 Drift

Trawt or dredge

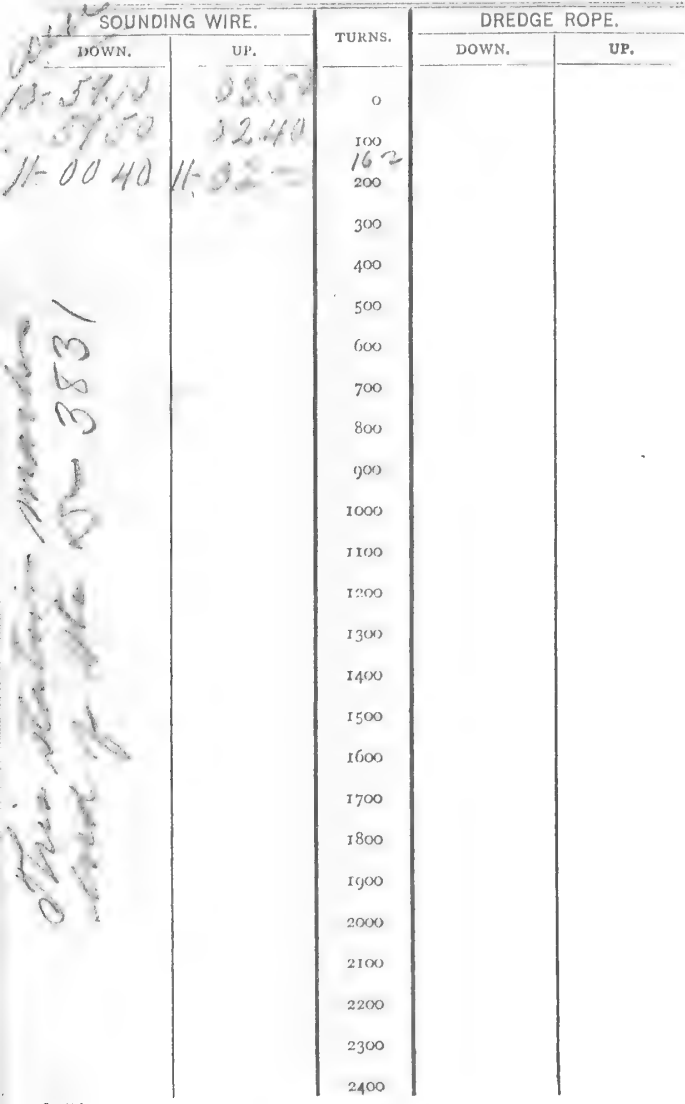




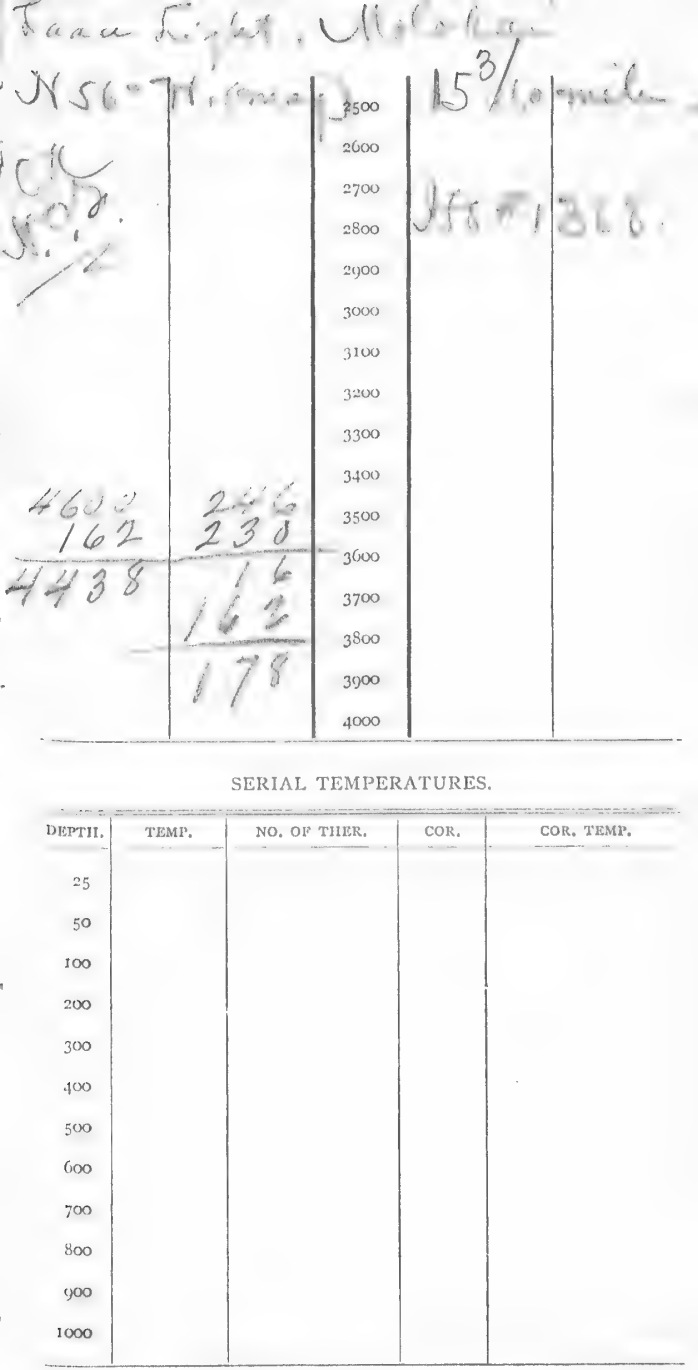

SERIAL TEMPERATURES

REMARKS : 
507.

(: No,

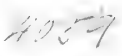

(Reel.

Therns / 2 Cor. +14 Depth

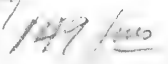

shatara lead

Botitom

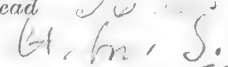

Bottom temperature

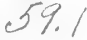

No. of thermometer $5048 /$ Cor.

-. Corrected temperature

Air 17 sierface 76 Drift

Trawl or aredise-

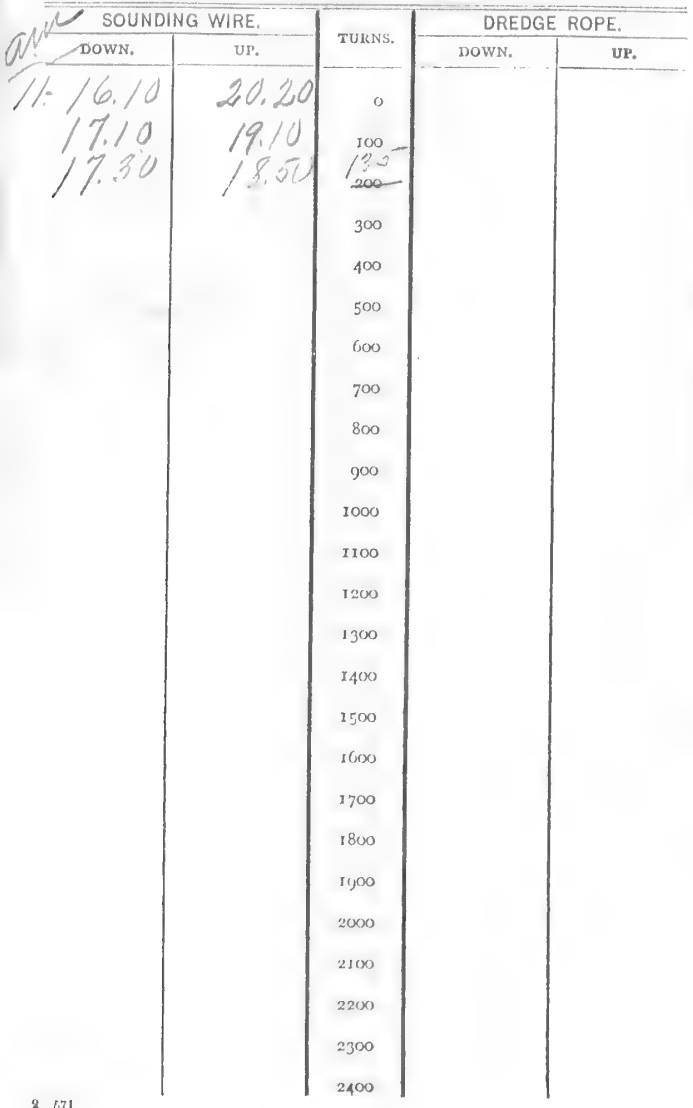




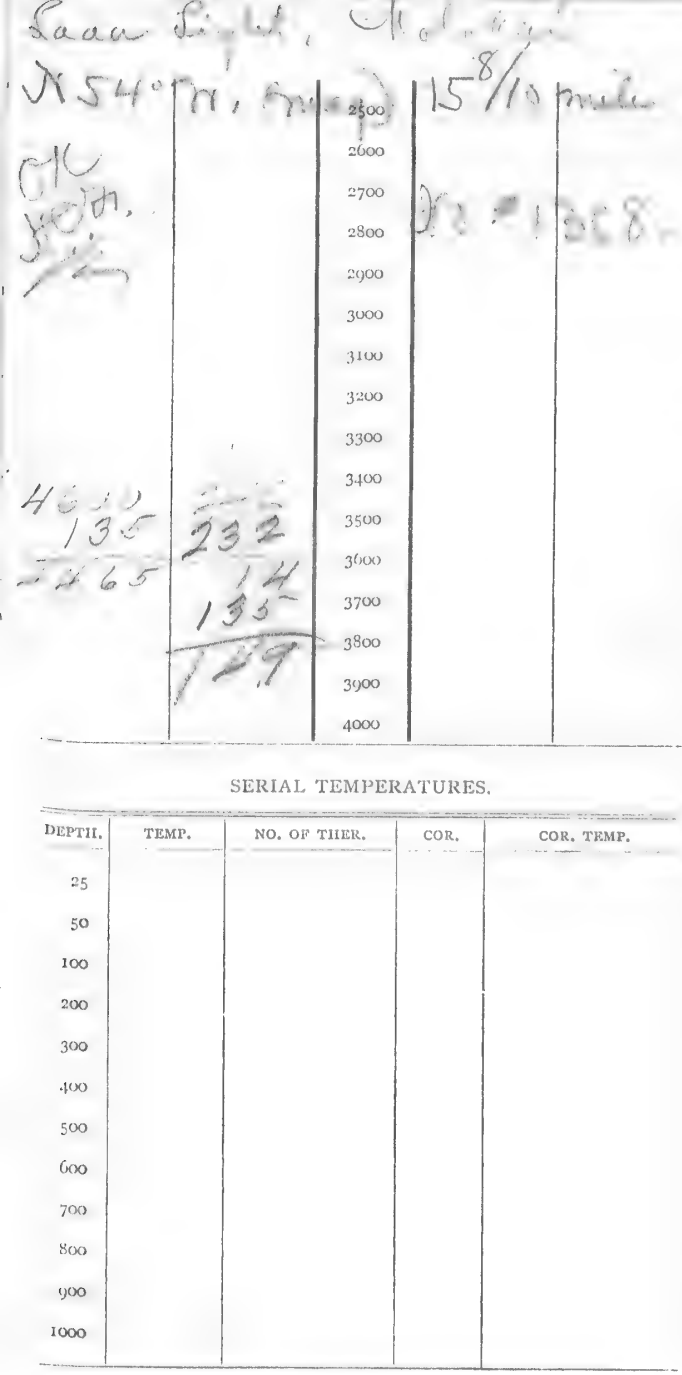

REMARKS : 
507.

Date

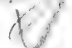

$\div 7.7$

No. 14y
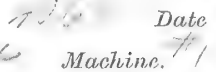

Reel.

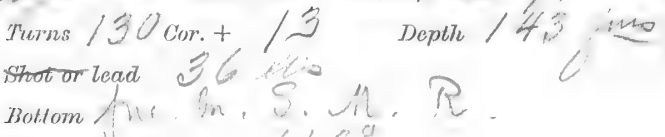

I3ottom temperabure

No. of thermometer

Cor.

Corrected temporature
Air
surfuce
Drift

Thaset or dredge

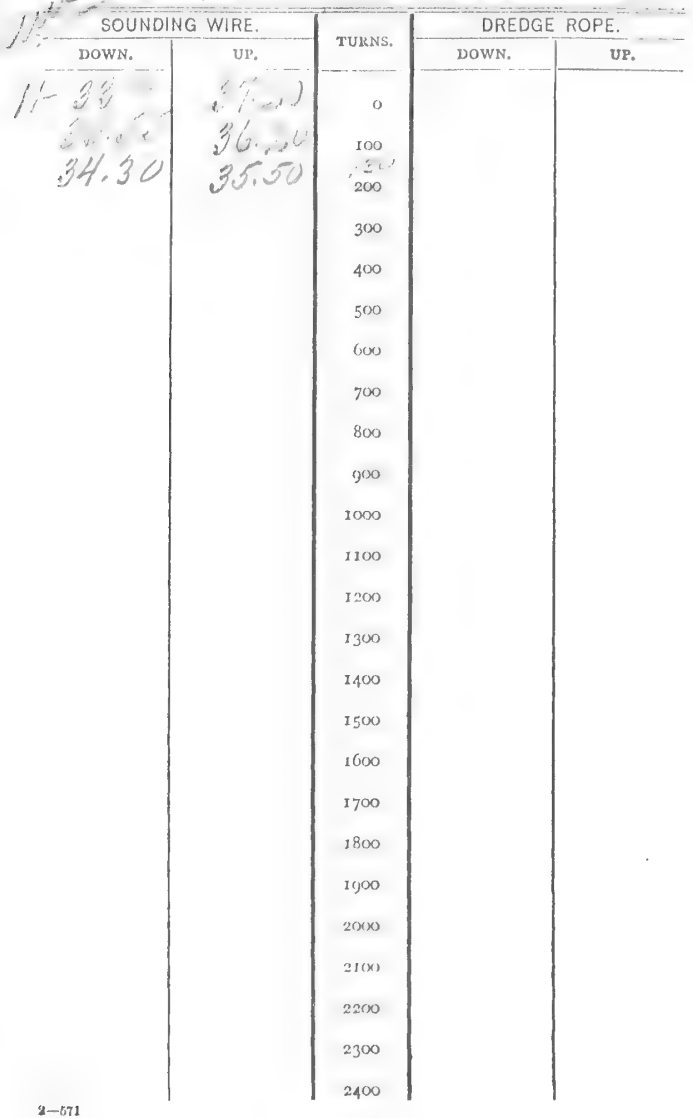




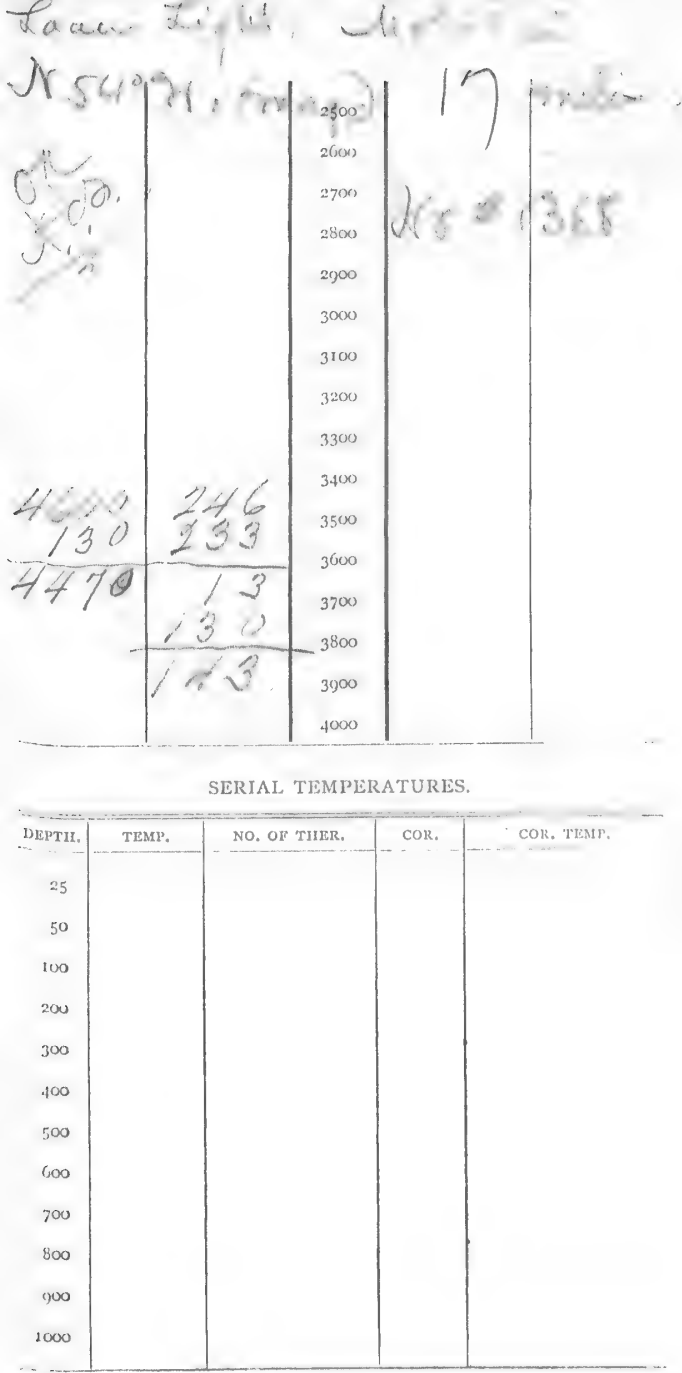

REMARKS : 
Tiurns $\therefore \because$ Cor. + Depth 7 sholigrileat, $A(s)$

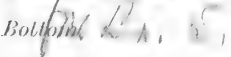
Bottom temperature

No. of thermotneter
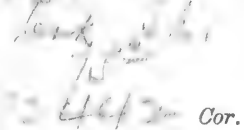

Corrected temperature
Air 11
surface
Trawl or dredge

$$
\because / 1 . \quad \text { Inrift }
$$

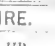
SOUNDING

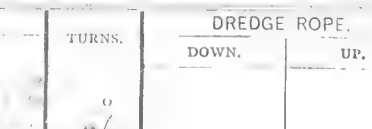

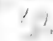

i

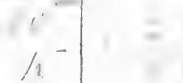

I $A(x)$

$15(x)$

1000 


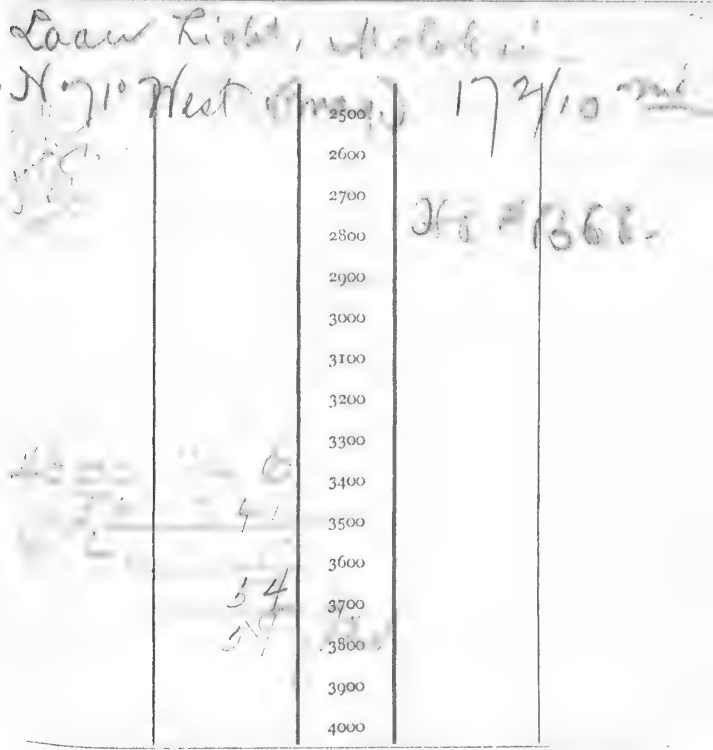

SERIAL TEMPERATURES.

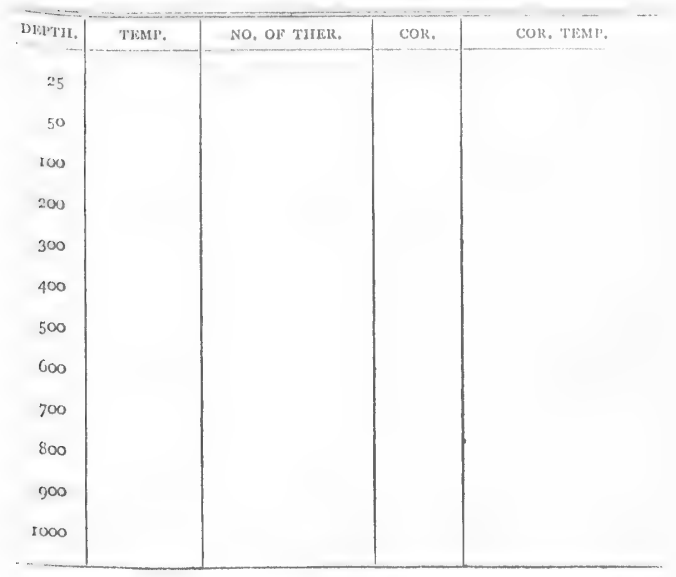

REMARKS : 
Aro.

Shot or lead

joltiom $\mathrm{HL}$<smiles>C=CO</smiles>

Depth 103$\}$ i ill

\section{Pottom}

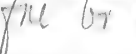

$\sqrt{1}$

Bottom lemperature

No. of thermometer

Cor.

Corrected temperaturi

Air 76 Surface $7 \%$ Drift

Irawl or alredge

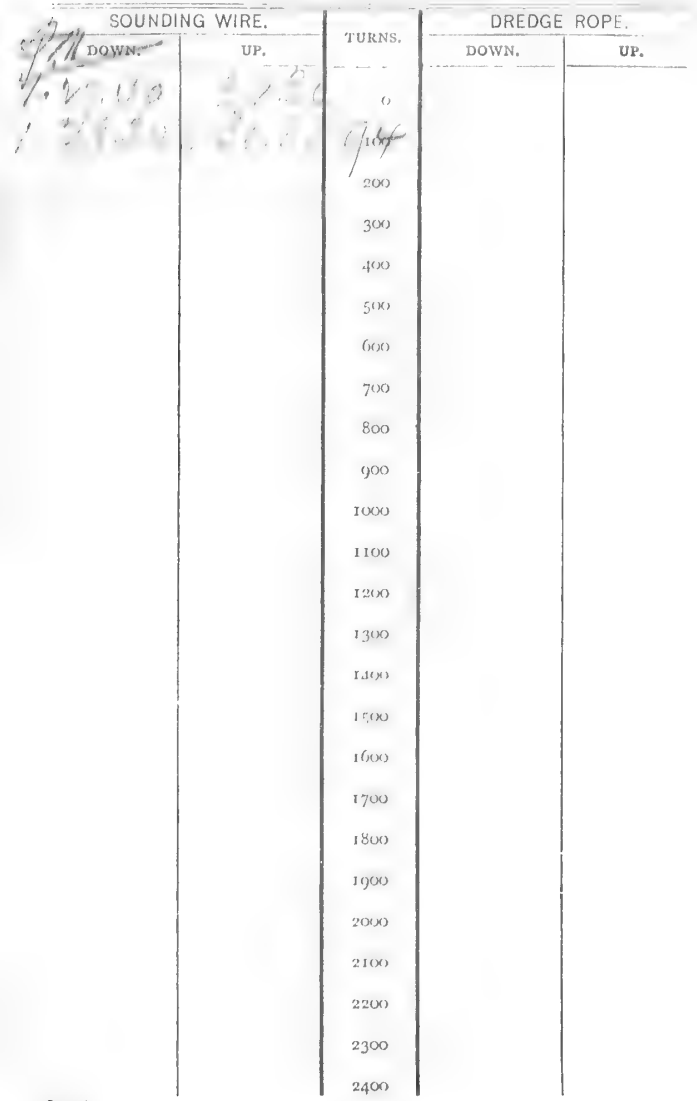




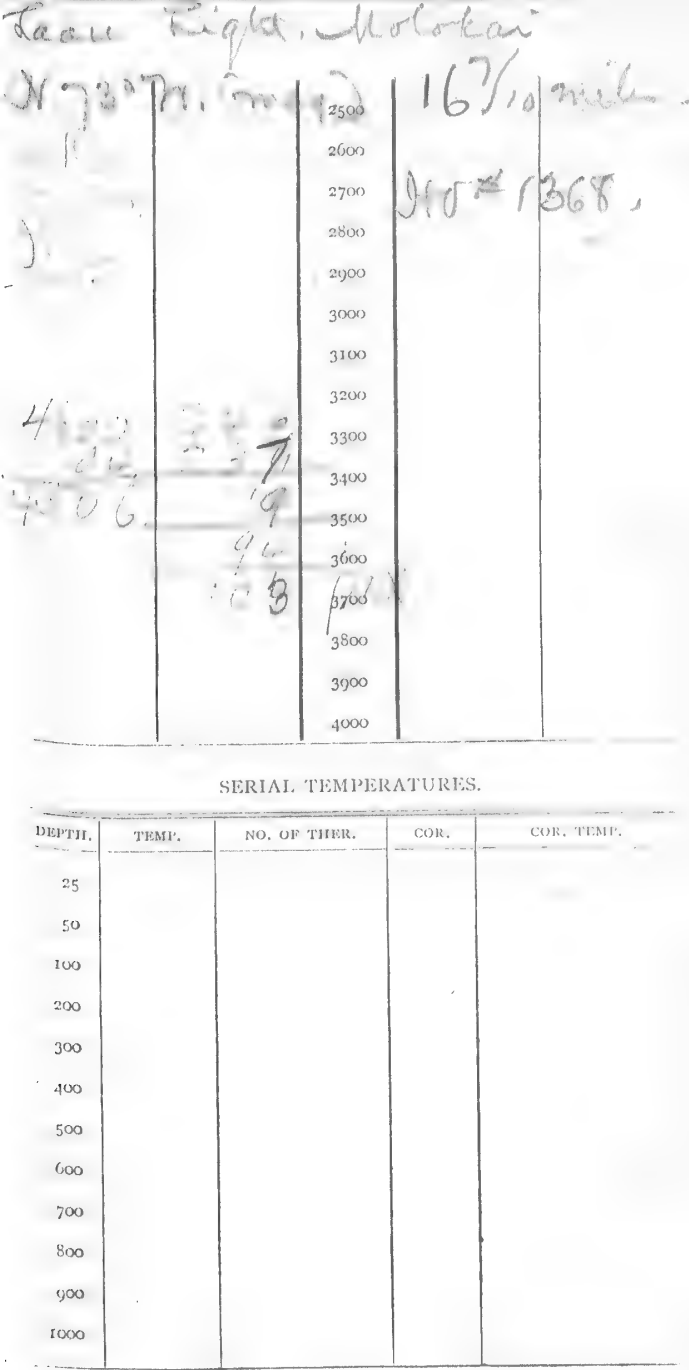

REMARKS : 
No.

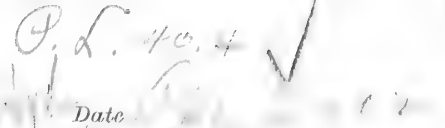

$\therefore$

Machins: Reel.

Turros

Cor. +

Depth

Shot on Zeaid

Bottom

Bottom temperature

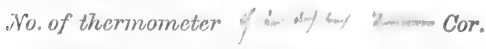

Corrected temperature

Air

Siurfoes

Drift

Trawl or aredge

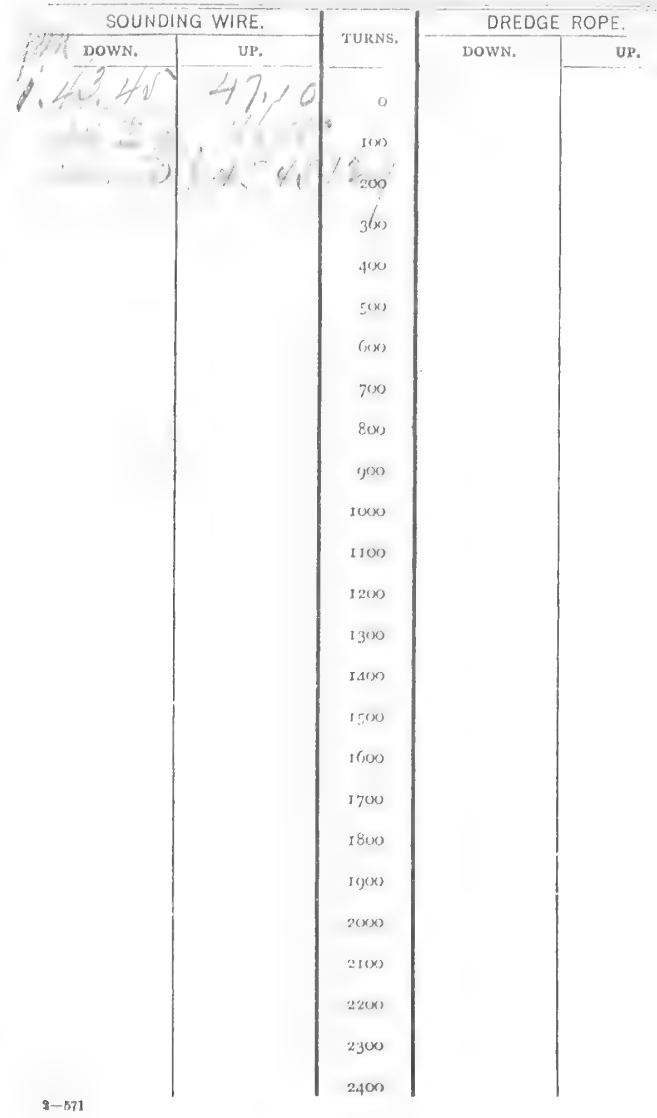




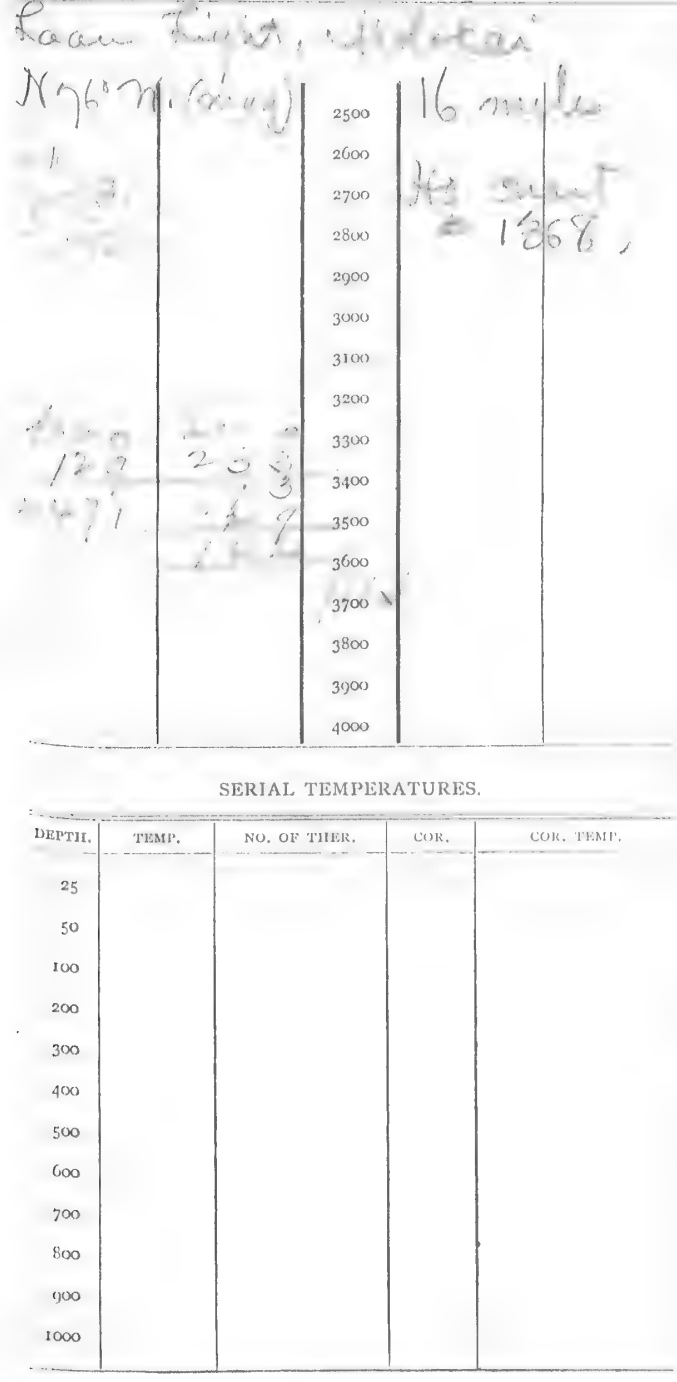

REMARKS : 
1) उorit

No.

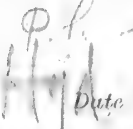

$\therefore 1,4$

Mrachille.

leel.

turns $/$ Cor.t

Depth

Shoobor lead/

Bottom
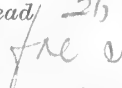

$\sqrt{2}$

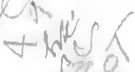

Bollom temperature

No. of thermometer

Cor.

Corrected temperature

Air . Surface

Drift

Trawt ór dredige

SOUNDING WIRE.

a Thowis.

in

Ci.

$\therefore, 0,0.0$

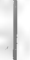

$1: 1$.

200

3(x)

fors

$5(x)$

G(x)

700

800

$(y)$

I000)

1100

I: $:(5)$

1300

I. $1(x)$

1500

I 6000

1700

I 800

1000

$20(x)$

$210 x$

2200

23000

$24(x)$

DREDGE ROPE. DOWN. UP. 


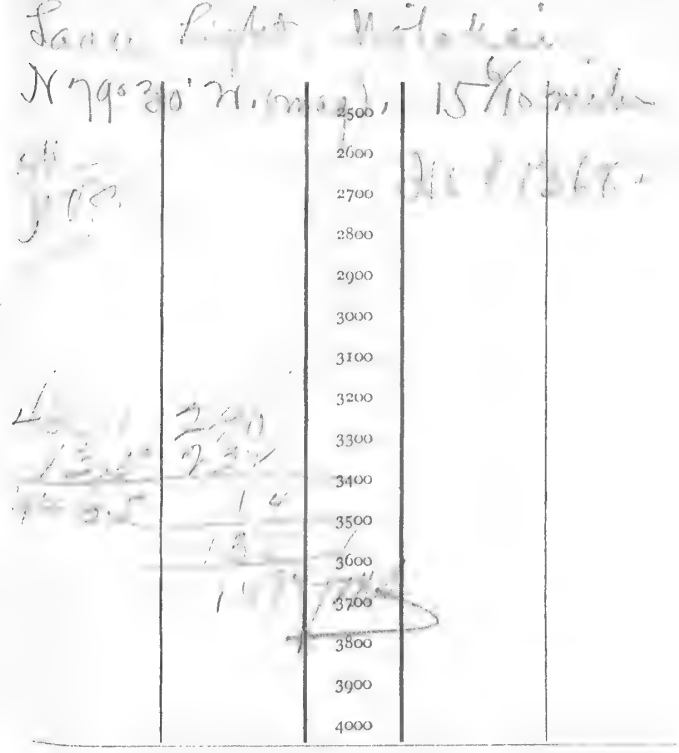

SERIAI, TEMPLRATURES,

\begin{tabular}{|c|c|c|c|c|}
\hline DEPTII. & Tresr. & No. OF THLR. & COR. & COR. THMF". \\
\hline 25 & & & & \\
\hline 50 & & & & \\
\hline 100 & & & & \\
\hline 200 & & & & \\
\hline 300 & & & & \\
\hline 400 & & & & \\
\hline 500 & & & & \\
\hline 000 & & & & \\
\hline 700 & & & & \\
\hline 800 & & & & \\
\hline 900 & & & & \\
\hline 1000 & & & & \\
\hline
\end{tabular}

REMARKS : 
No. 3622 Date

Machine./ Reel.

rurns / Cor: +

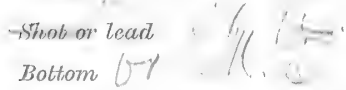

Depth $A S^{1} \mathrm{~B}$

153

Bottom temperature

No. of thertnometer

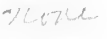

$7+4 \hat{1}$

Cor.

Corrected temperature

Air

Sictrayes

$\rightarrow 6$

Drift

Trawl on dreadege

SOUNDING WIRE.

DOWN. UP.

$\log (1)+3 \ldots$

at $/ 1$ it

$2 y 100$

$1 ., 50 C$

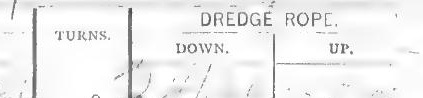

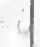

$$
0
$$

100

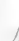

1

$\frac{1}{2}$

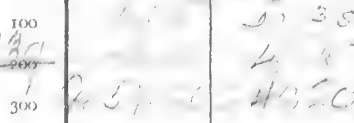

$4(x)=$ if

$f(x)$

700

$8(x)$

$y(x)$

I(i) ()

11100

I 200

J 300

$I \triangle O O$

1500

1600

1700

1800

1 gon

2000

2100

2200

2300

$24(x)$ 
เom.

$\therefore$ No

\section{Date}

Machine:

Reel.

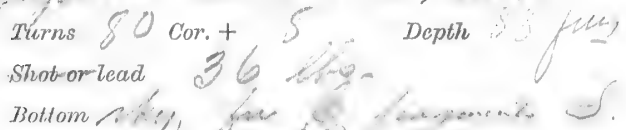

IBottom temperature

No. of thermometer

Cor.

Corrected temperature

Air 76 surface $\%$ Drift

\section{Trawi-or-ilrailge.}

\section{(i) SOUNDING WIRE.}

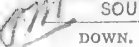

$3-2 \% 31$

$2, i(1)$

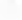
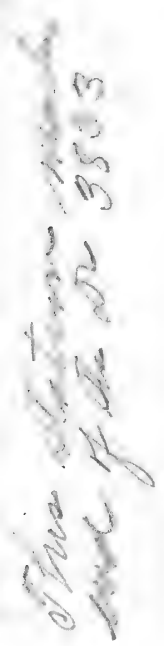

$8-571$

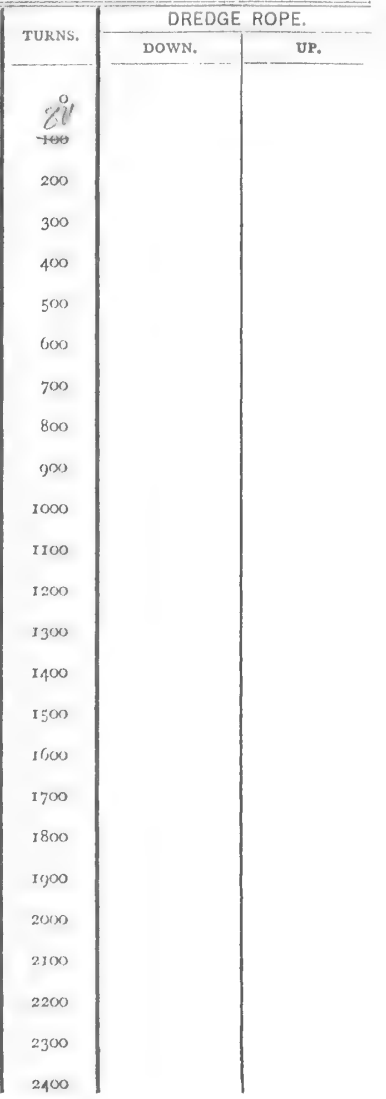



507.

1 .

No:

Date

$C \therefore \therefore \div 2$.

Mashine. - Reed.

Therns - Cor. + - Depth \& $\cdots$.

shoot-or lead

Bottom

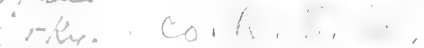

Bottom temperature

No. of thermometer

Cor.

Corrected temperature

Air $7 \%$. surface. $7 \%$ Drift none.

Twartar ar dredge

SOUNDING WIRE.

DOWN.

up.

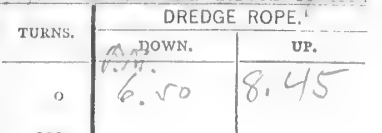

200

300

400

500

600

700

800

goo

1000

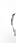

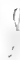

nes ode

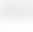

1100

1200

1300

1400

iา,

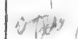

1600

1700

1800

1900

2000

2100

2200

2300

2400 
No.

Machine.

Thurns Cor.t Depth

Shotion lead

Bottom

Bnttom temperature

No. of thermorneter

Corrected temperature

Air

Surface

corr.

Trawl or dredge

\begin{tabular}{c|c|c|c|c}
\multicolumn{2}{c|}{ SOUNDING WIRE. } & DREDGE ROPE. \\
\hline DOWN. & UI. & TURNS. & DOWN. & UP. \\
\hline
\end{tabular}




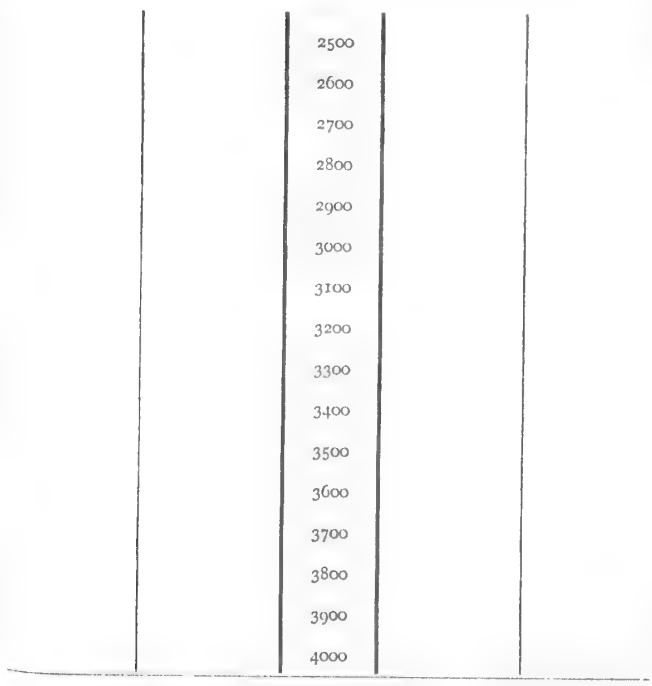

SERIAL TEMPERATURES,

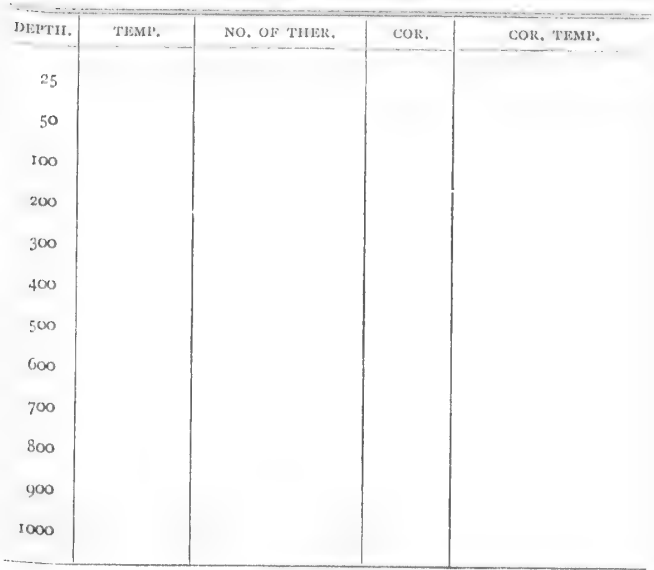

REMARKS : 
$50 \pi x^{2}$

No. 1066

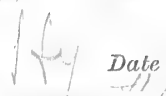

Machine.

Turns:L. Cor +1 if Depth $\%$

Shatuatialead

Bottom

Brttom temperature

No. of thermometer

Cor.

Corrected temperature

Air $>\div$ Surface $\%$ Drift

Trautl or dredge

SOUNDING WIRE.

DOWN.

U1.

TURNS.

DREDGE ROPE.

$+1,1 / 1000$

-

DOWN.

UP.

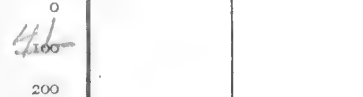

300

400

500

Goo

700

800

goo

1000

1100

1200

1300

I 400

1500

1600

I 700

1800

I goo

2000

2100

2200

2300

2400 


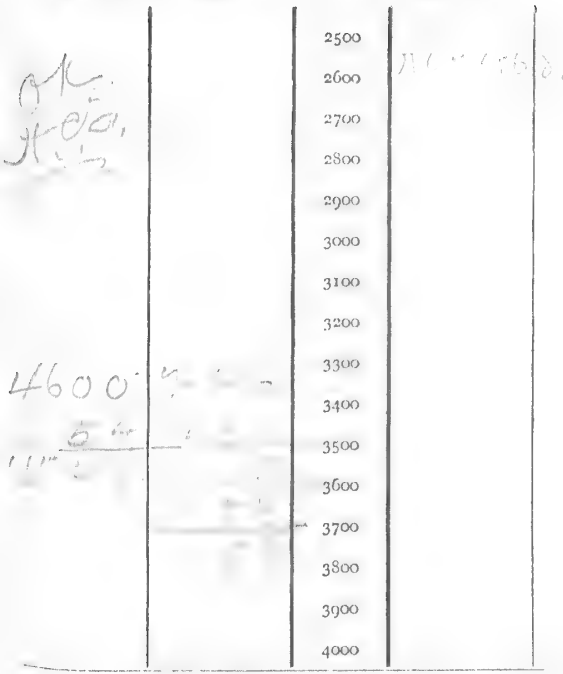

SERIAL TEMPTERATURES.

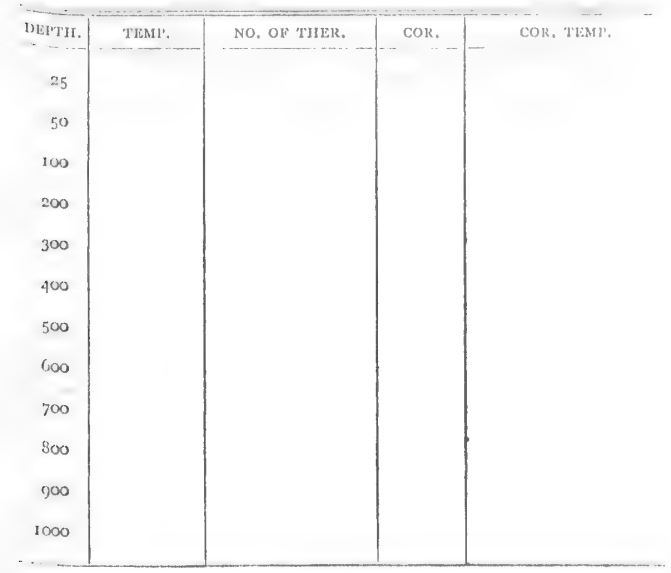

REMARKS : 


$$
\frac{11}{11}
$$





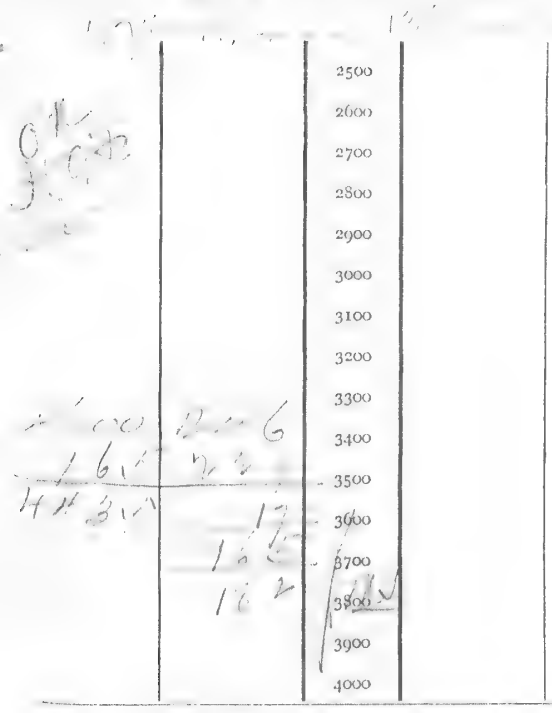

SERIAI, TIMI'RATURIES.

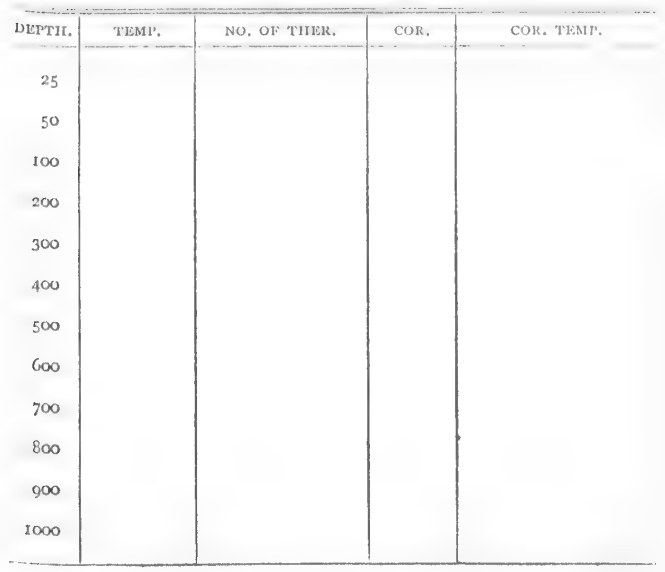

REMARKS : 


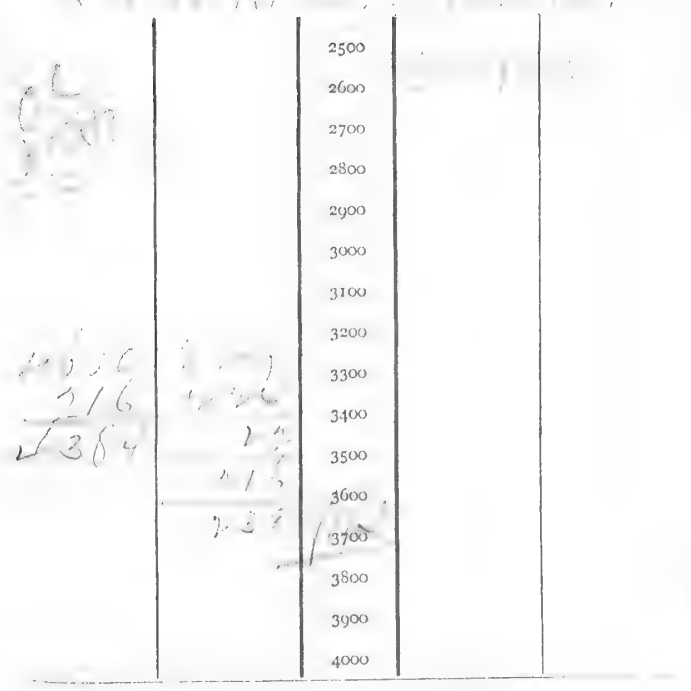

SERIAL TEMPLRATURES.

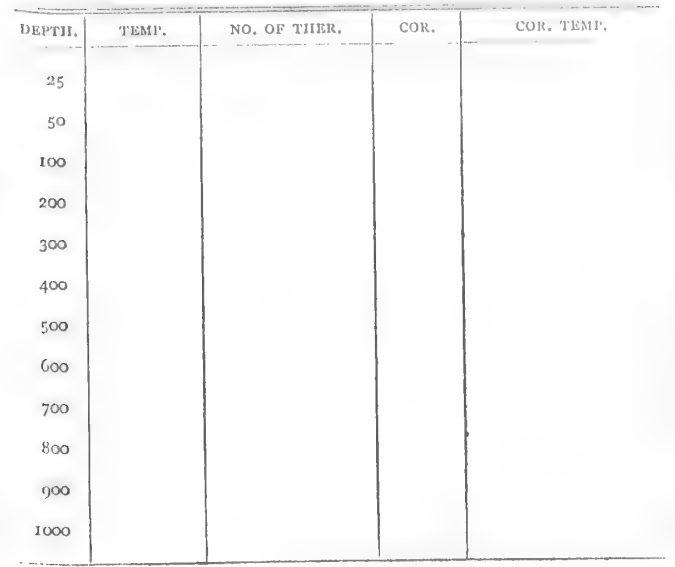

REMARKS :

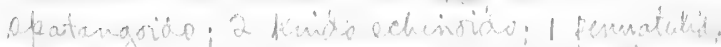

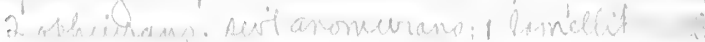


No. thy \&ubor Date -

Tanner Machine. Reel.

Turns 256 Cor +9 Depth $255 / 4$

szator lead $26 \alpha \mathrm{h}_{2}$.

Bottom oy lit.

Bottom temperature

No. of thermometer

Cor.

Corrected temperature
Air
Surface
Drift

Trawl or dredge

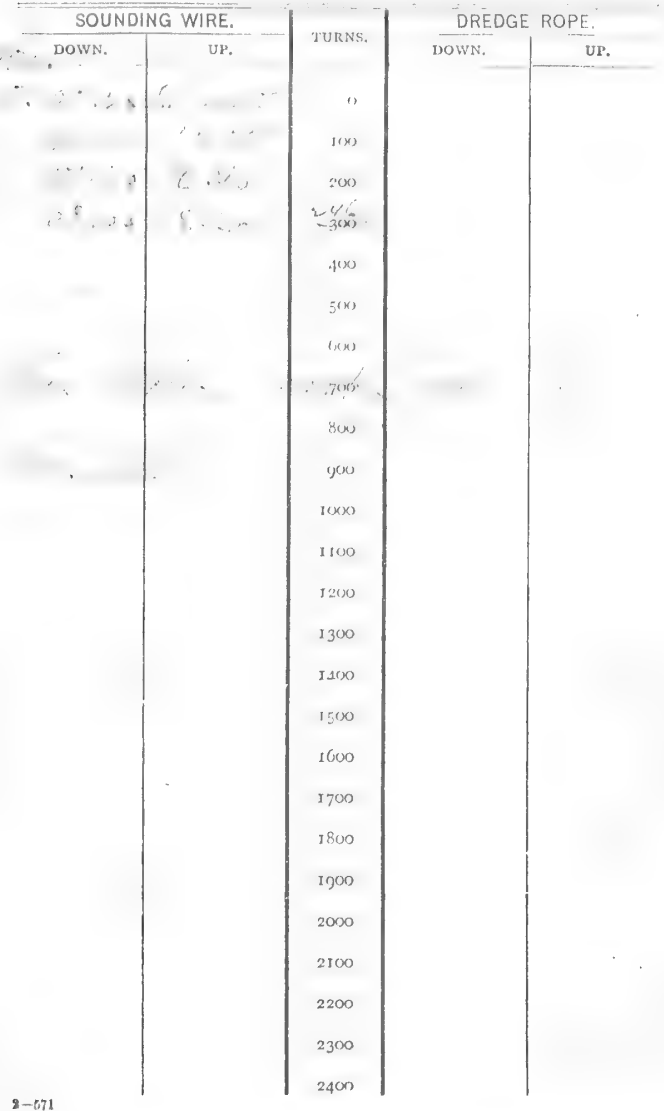




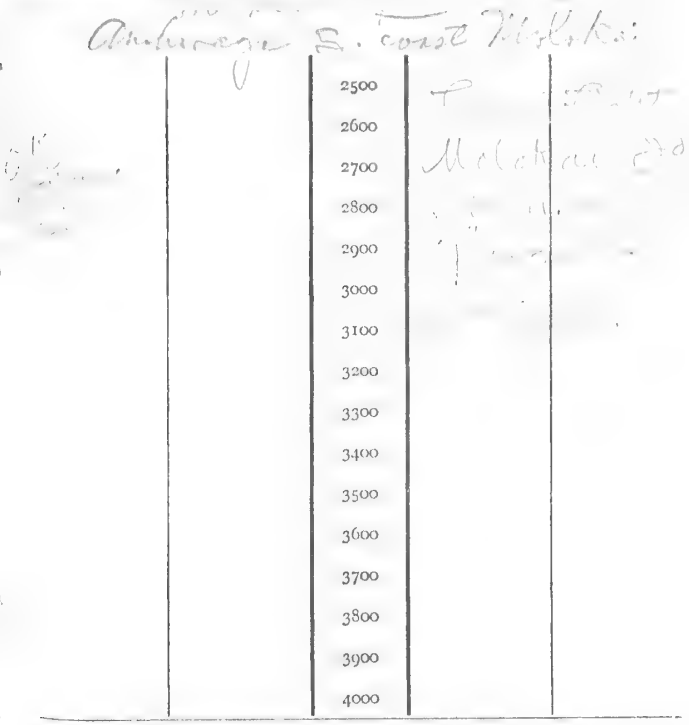

SLRIAL TEMI'ERATUIRES.

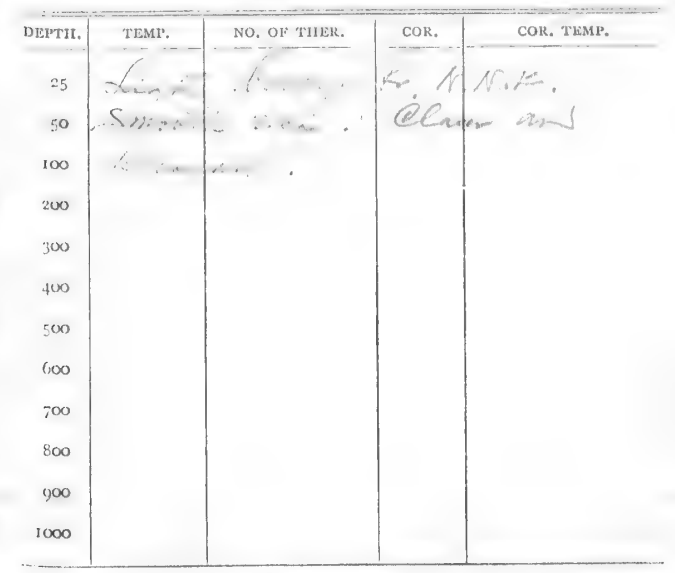

REMARKS :

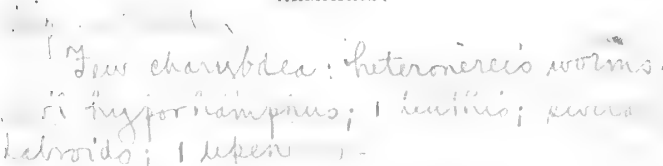




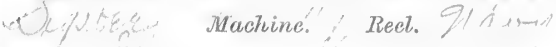
Turns
Cor. +
Dopth $8 / 5$
Shotina lead

Bottom gy, by

Bottom temperature

No. of thermometer

Cor. $\quad j+70\}$

Corrected temperature

Air 72 Sunface 75 . Drift

Tratomedise:

$\overline{\bar{f} \text { SOUNDING WIRE. }}$




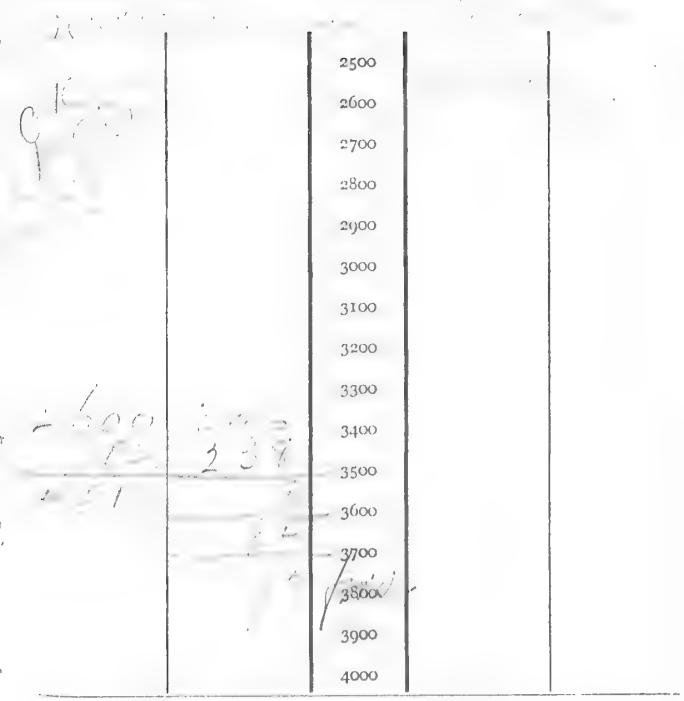

SERIAL TEMPERATURES.

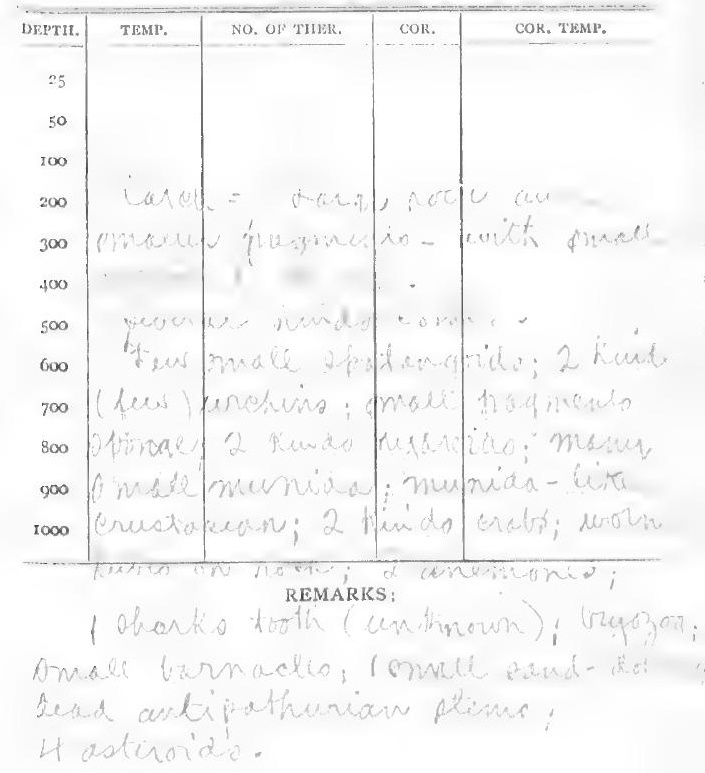




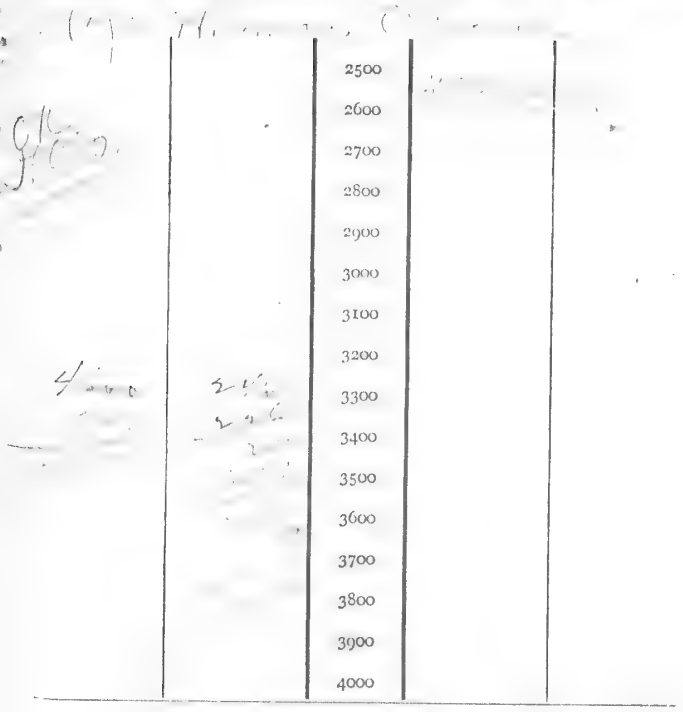

SERIAL TEMPERATURES.

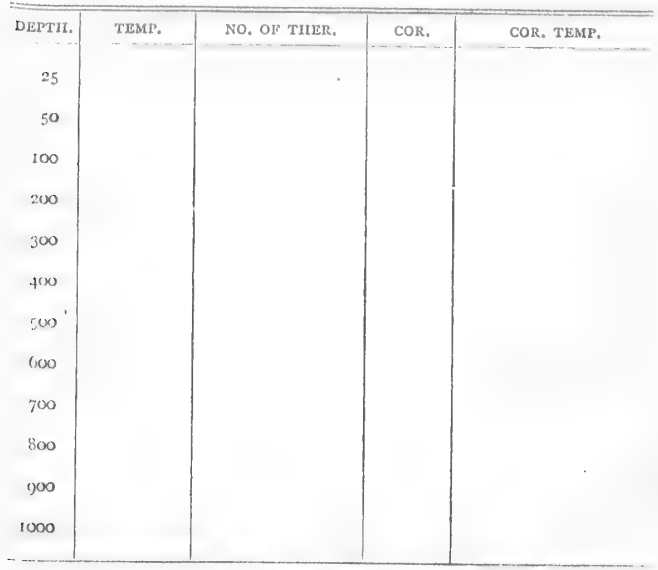

REMARKS : 
5077 .

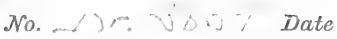
Sin -ace Machine /Reel.

Thorns 23 Cor. +

$2 \%$

Depth $\quad 2 \sqrt{7}$ fin

Shot or lead

Bottom $A, f r, v l, S$.

Bottom temperature

No. of thermometer

Cor.

Corrected temperature

$840^{2} 7$

Air

Surf face

Drift

Trawl ordrodge: Y i ears

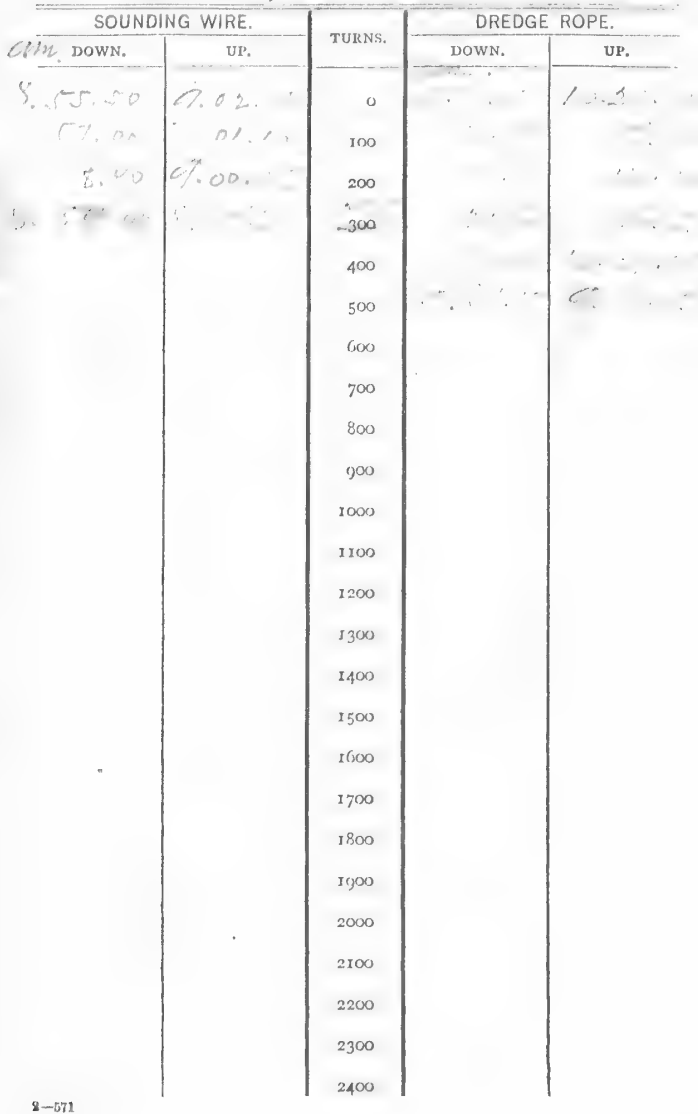




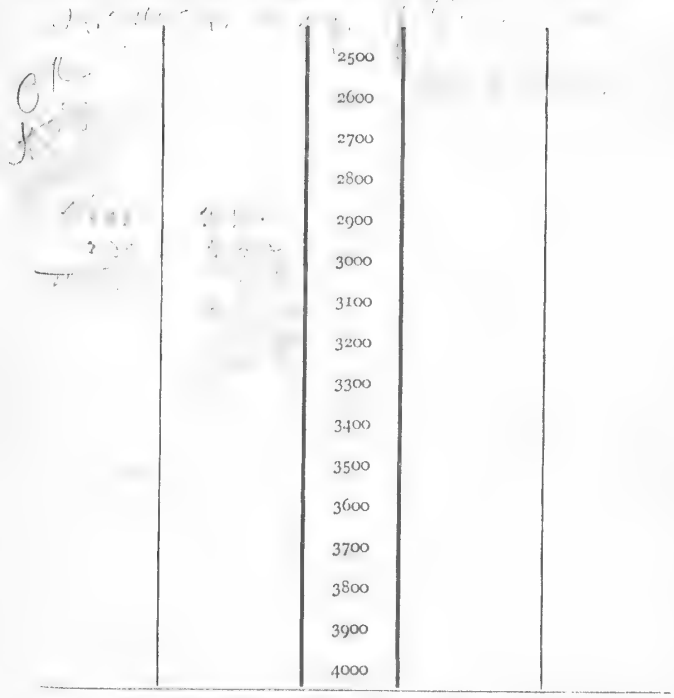

SERIAI, TIMPERATURES.

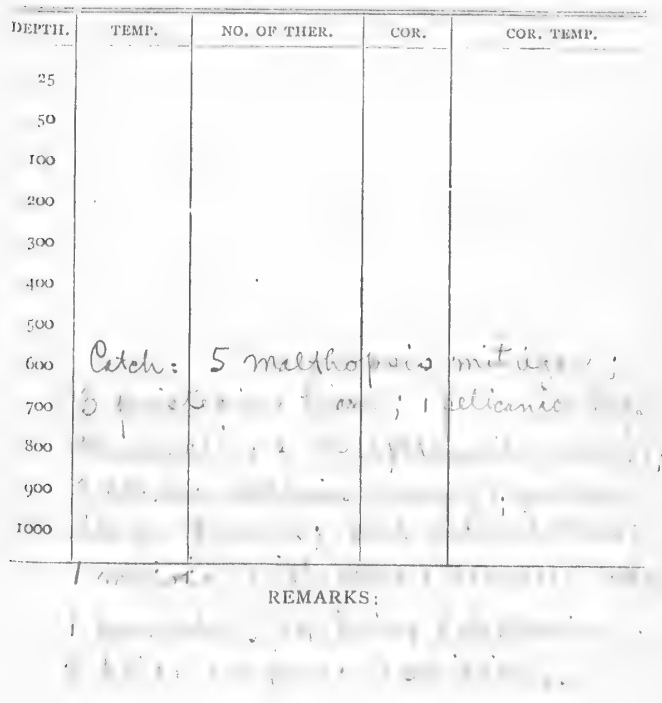




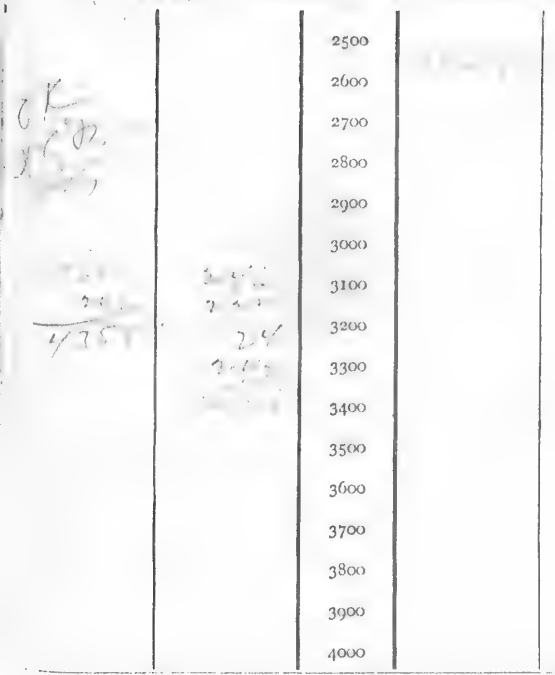

SERIAL TEMPERATURES.

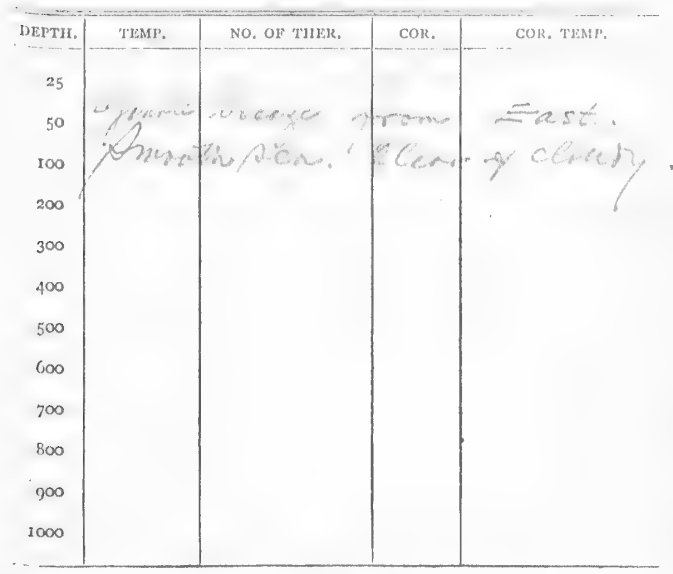

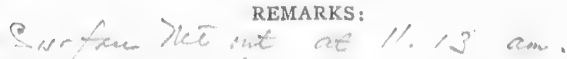

$$
\begin{aligned}
& \text { " in } 11.2 \%
\end{aligned}
$$

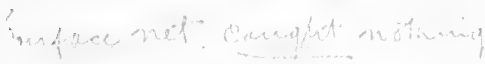

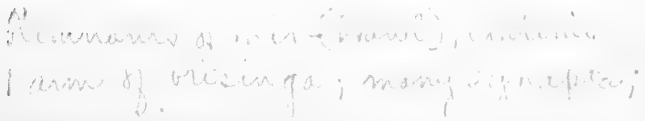


Who 강

\section{Date}

S Machine Red. A R ....

Turns 20 Cor. + Depth a $1, \cdots$

Shotor lead

Bottom

$56: 2,1,6$

Bottom temperature

No. of thermometer

"2)

8

Cor.

Corrected temperature

Air

Sierface

Trawt andredge ef $=132 .+\mathrm{c}$

Drift

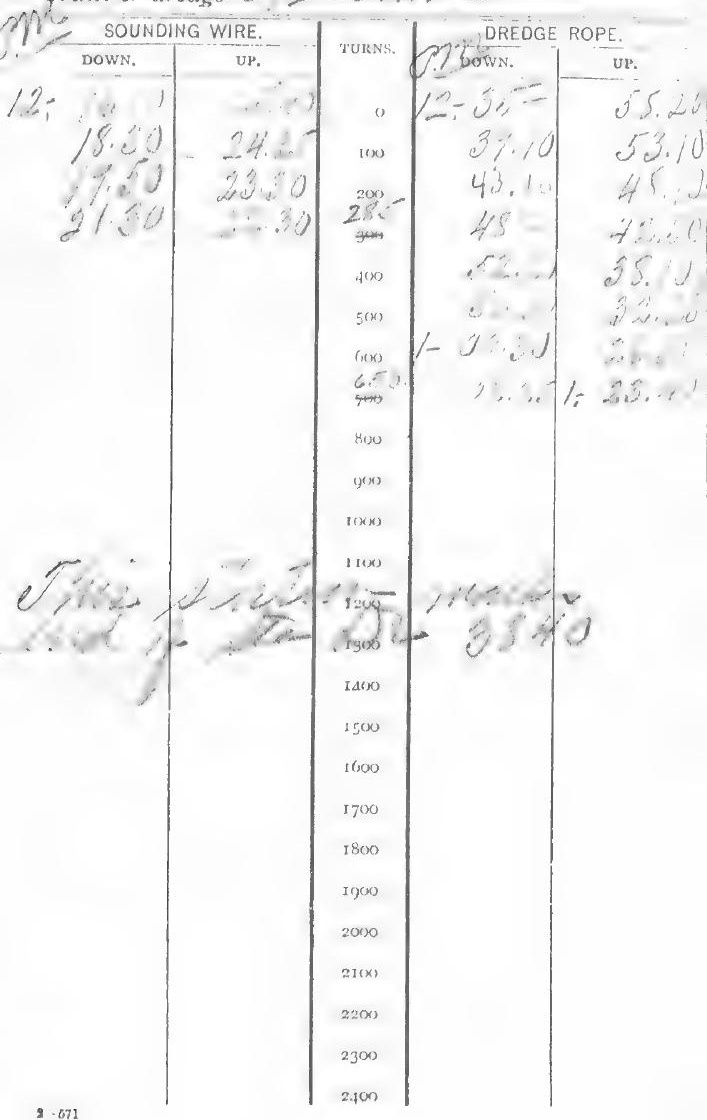




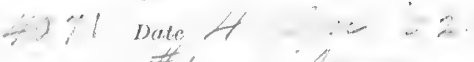

स-2wore Machino \#/Reel.

Turns t Cor. + Depth

shotorlead, क्ष 6 .

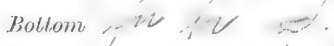

Bottom,temperature

No of therrometer

Cor.

Corrected temporature

Air $7 \%$ Drifface 76 Drift

Irawl or chredge

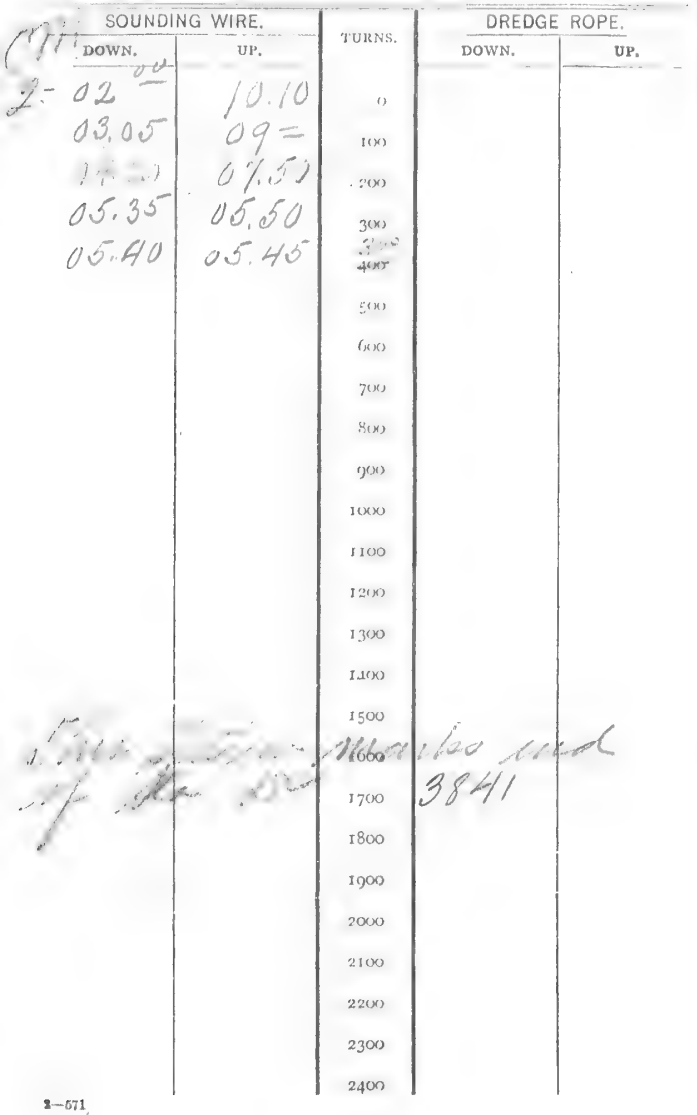




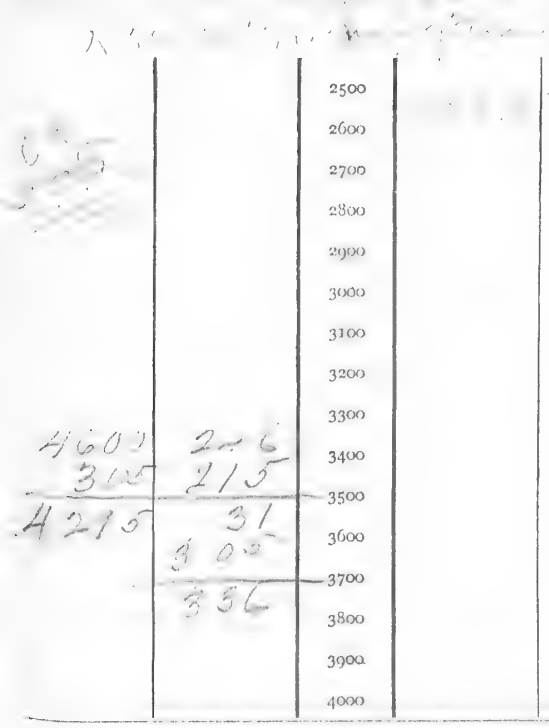

SERIAL. THMP'ERATURES.

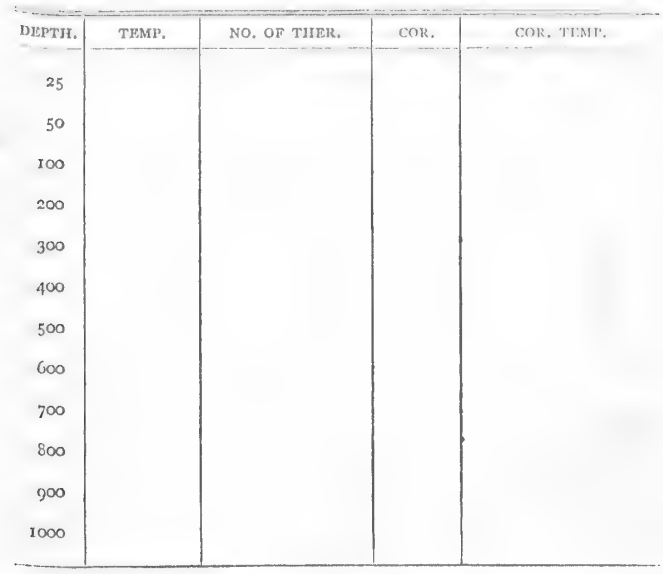

REMARKS : 
LC Machine. Thel.

Turns 70 OCor.

Shotror lead

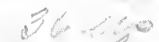

liobtom

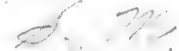

Bottom tenuperature

No. of thermometer

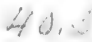

Cer.

Corrected temperature

Air 7 Surface

Drift

Trawl or aredse

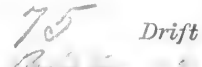
$\because$, SOUNDING WIRE.

DOWN. $\therefore 5,50$ ४) 分方, ” $\{+\cdots$ $\therefore, ?$ $i$ UP. 46.56 $\therefore$

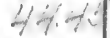
13. $\because \div$ $\because 2 \cdots=$ $\because 2$ TIDAREDGE ROPE

(n)

700

sou

$12(x)$

1300

I A (X)

5500

1600

3700

1800

Inoro

2000

2100

2200

2300

2400 UP.

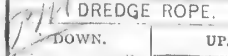
C. TOWN. $j+11,0$ i. -21 ),-1

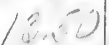
17.30 $1 / 10$ 05.1 


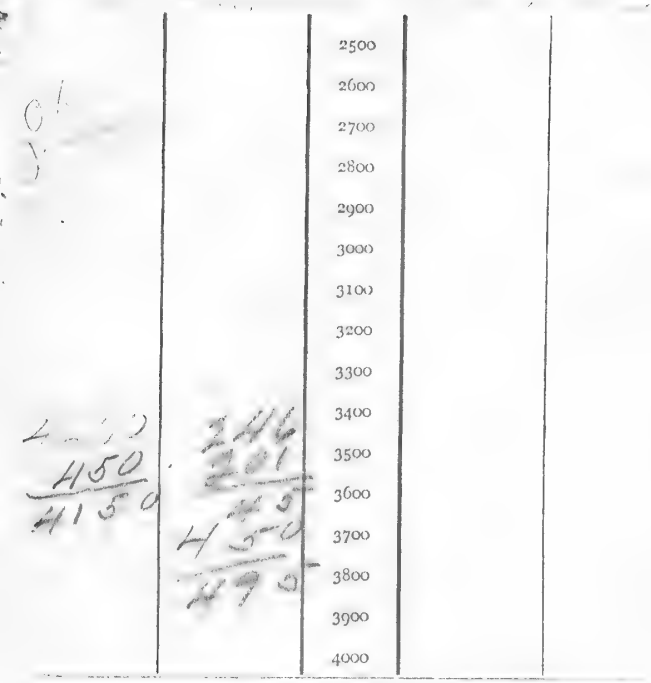

SERIAI, THMI'ERATURES.

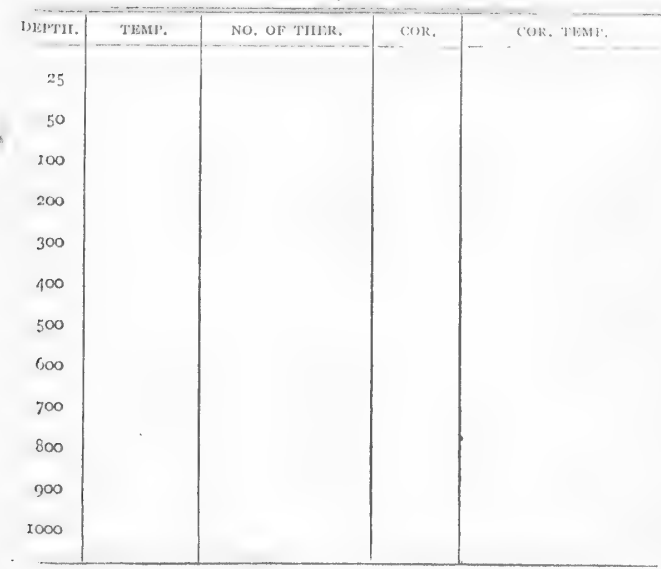

REMARKS : 


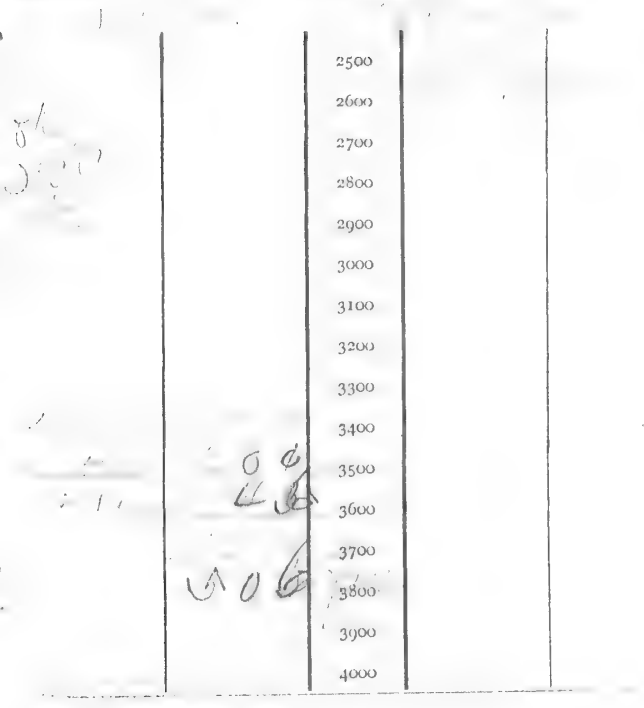

SERIAL TEMPERATURES,

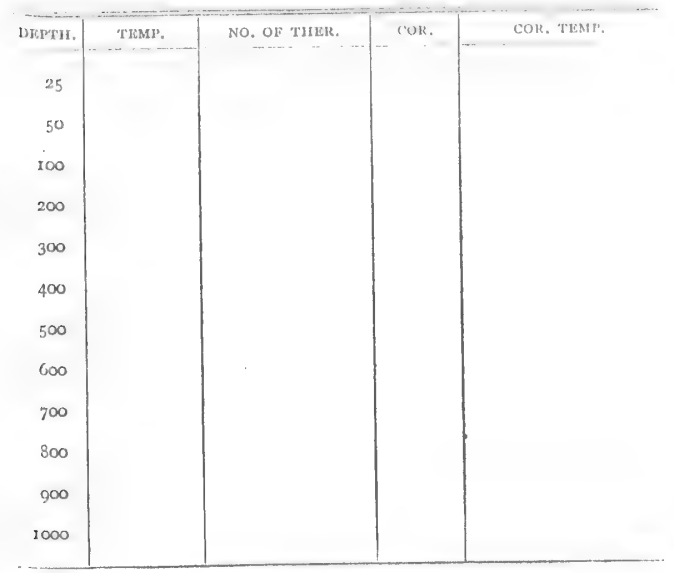

REMARKS : 
Machine..... Reel.

Turns

$$
\text { Cor. }+
$$

Depth $/ 71^{-14}$

-Shot on lered

\section{Bottom}

Bottom temperature

No. of thermometer

Cor.

Corrected temperature

Air 72 Surface

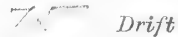

Trawl or dredsc

\section{SOUNDING WIRE.}

DOWN.

UP.

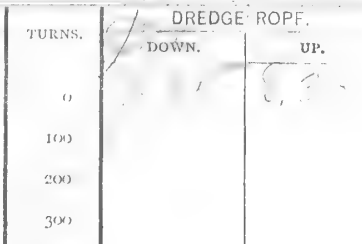




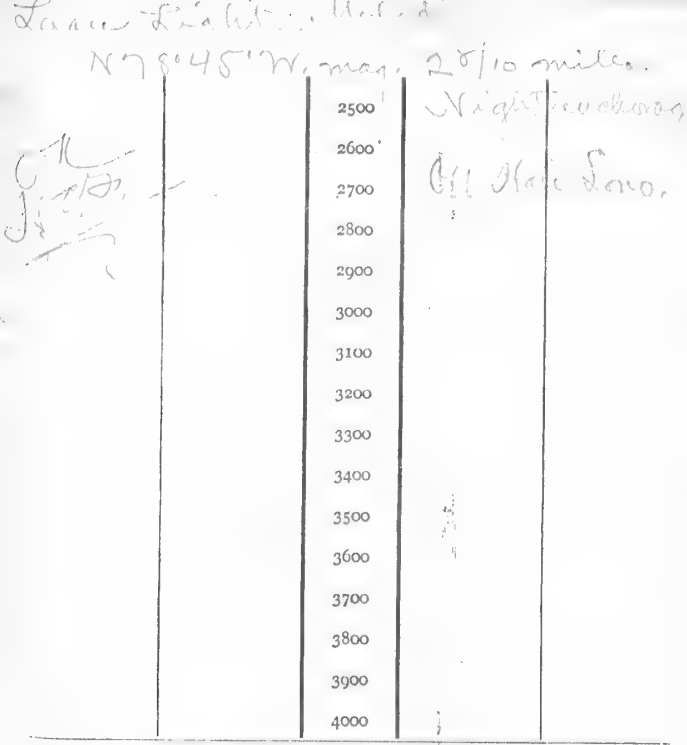

SERIAL TEMPERATURES.

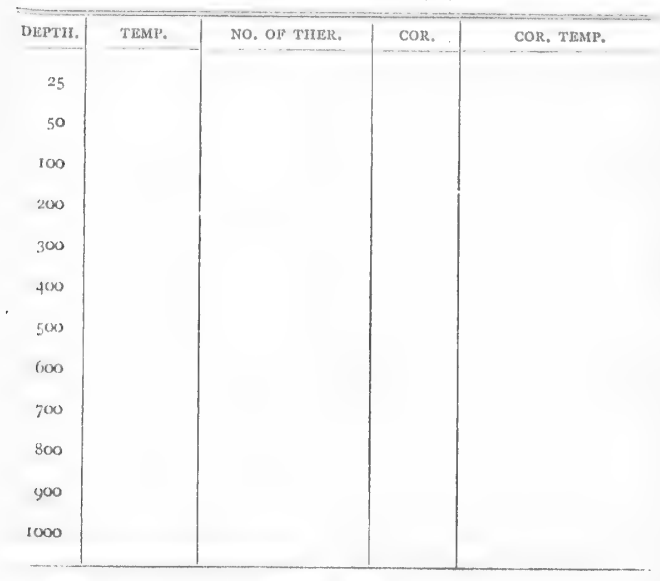

(1) REMARKS : 
Machine. 'Reel.

Therns

$$
\text { Cor. }+
$$

Depth

Shot or lead.

\section{Bottom}

I3rttom temperature

No. of thormometer

Corrected temperalure

Air $7 ?$

Surface

- Trawl or dredre

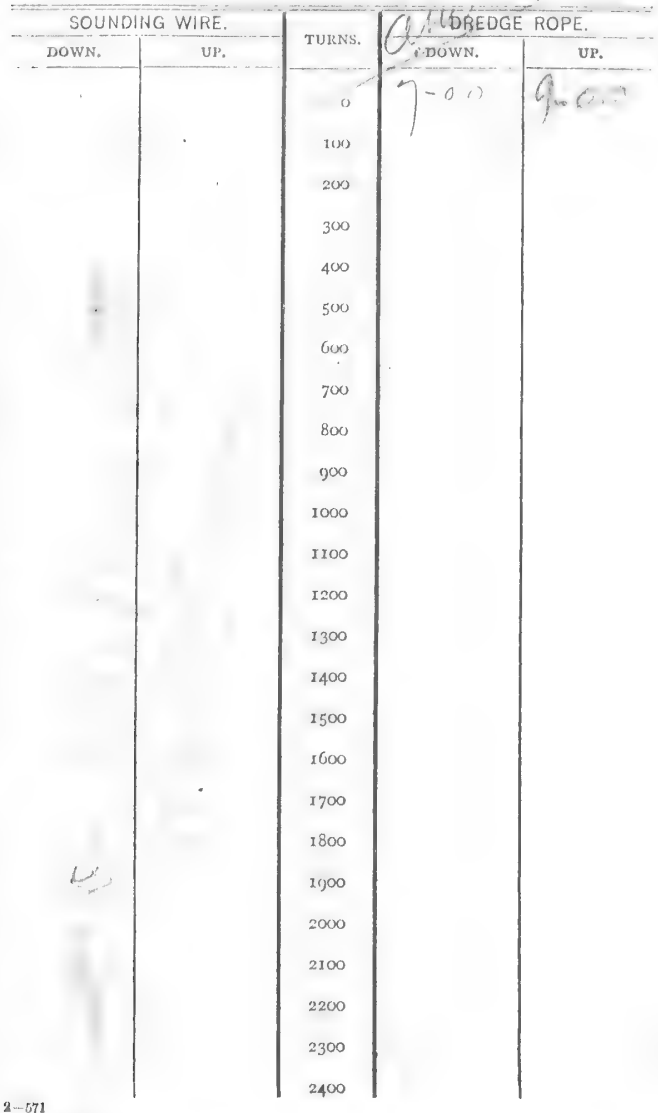




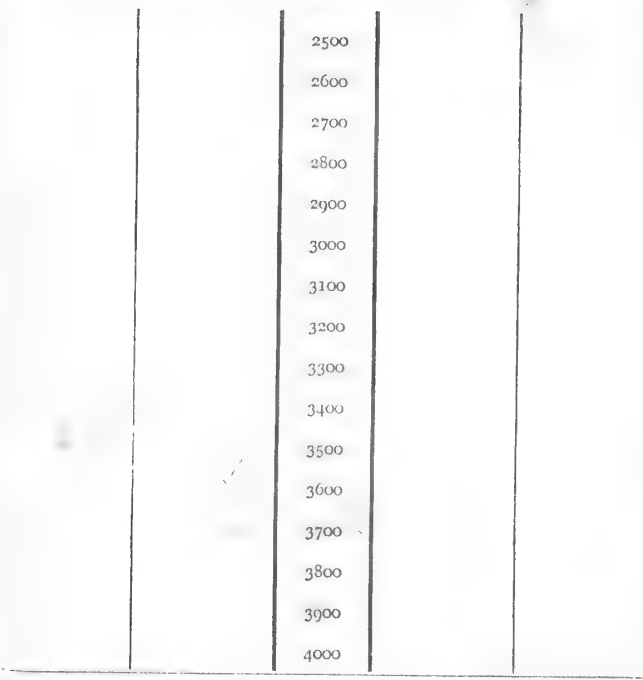

SERIAL TEMPERATURES.

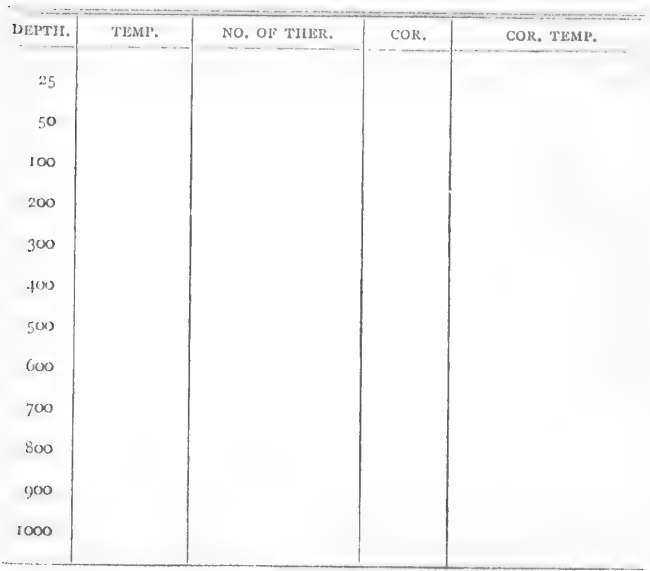

REMARKS : 
507.

Jyo.

Date

\section{'Machine. Reel.}

TuTns

$$
\text { Cor t }
$$

Dcpth

shot or lead

Bottom Gro, l?

Brittorn temperature

No. of thormometer

Cor.

Corrected temperature

Air

Irawt or dredge

$$
\text { Surface } 7)
$$

SOUNDING WIRE.

$$
\text { DOWN. }
$$

UP.

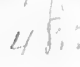




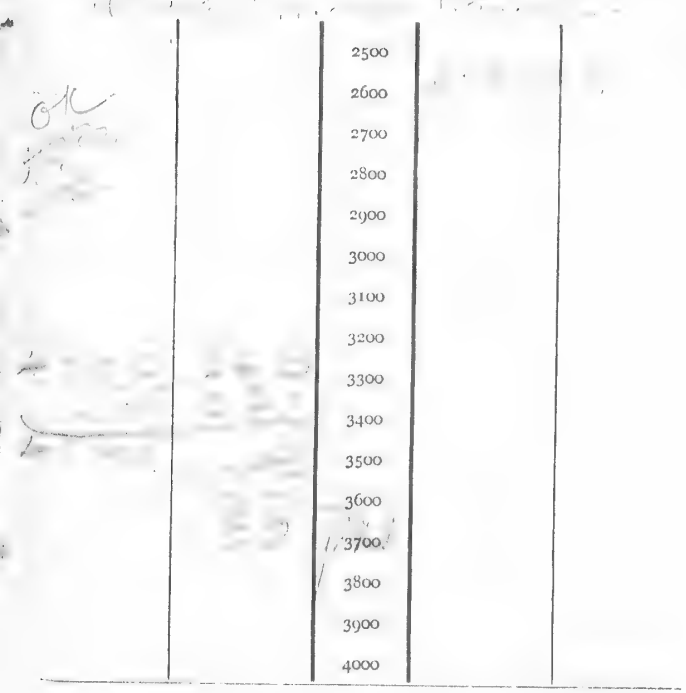

SERIAL TEMPERATURES.

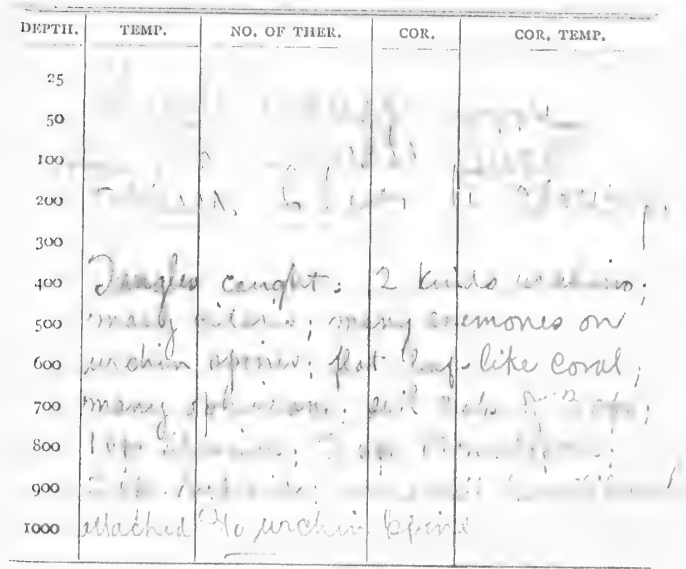

\section{REMARKS :}

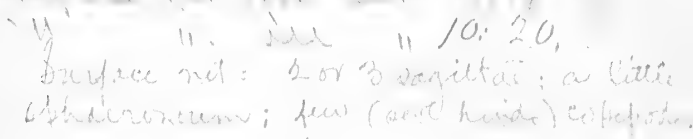


$m$.

No. 2 Date

(p) Machine. /, Reel.

Turns Cor. +

Depth

S7zot.or lead

Bottom cro. fr. S.

Bottom temperalure

No. of thermometer
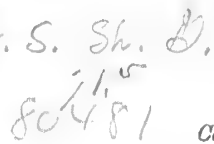

Corrected temperalure
Aiv
Surface
Drift

Trawl or-dredge

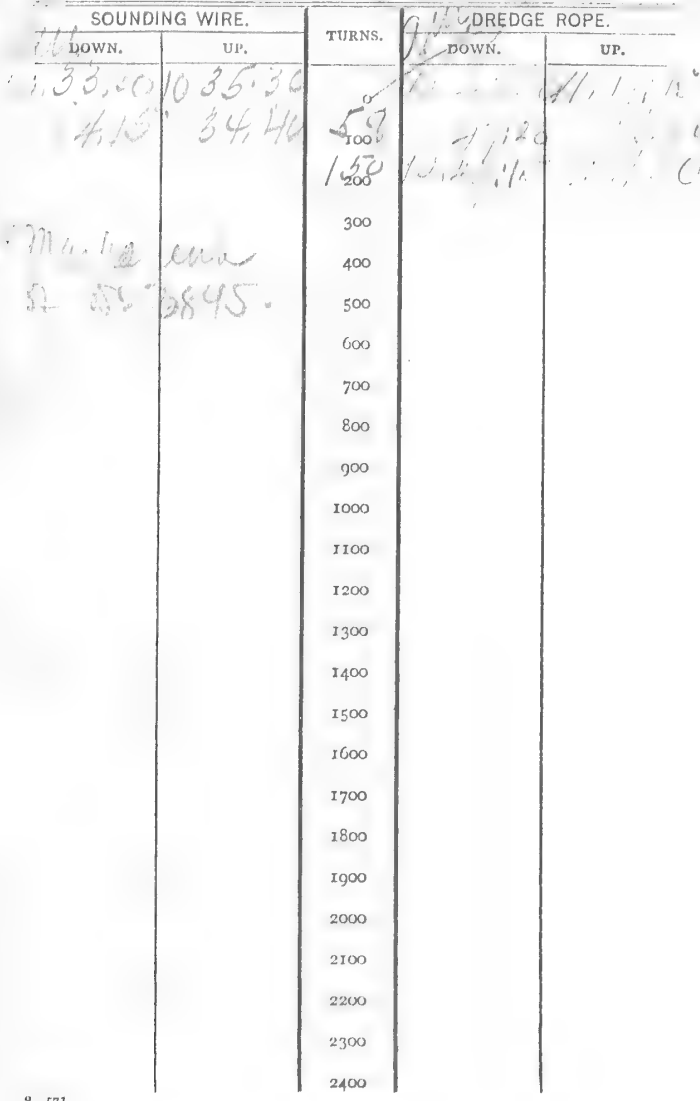




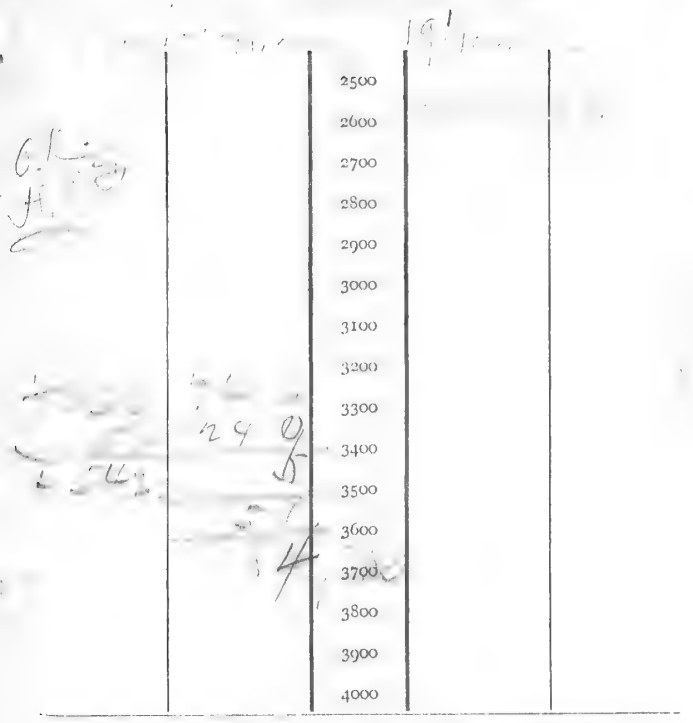

SERIAL TEMIJIRATURES.

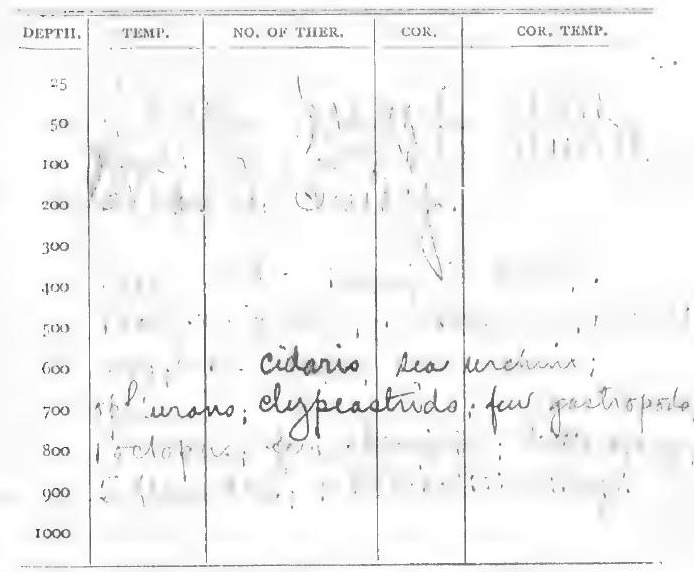

REMARKS:

O)W

11 i1: 11

$\therefore$. . : $\div 4$ 
foon.

so. 11073

Machine. $/$ Reel.

Nater

\section{5}

$\mathrm{Cor}_{4 ;}+$

5 Depth

Shat or lead

Bottom

Bottom temperature

No. of thormometer

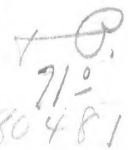

Cor.

Corrected temperature

Air 76 Surface 76 Drift

Trawt or dredge

\begin{tabular}{|c|c|c|c|c|}
\hline \multirow{2}{*}{\multicolumn{2}{|c|}{$\begin{array}{l}\text { SOUNDING WIRE. } \\
\text { OWN. }\end{array}$}} & \multirow[b]{2}{*}{ TURNS. } & \multicolumn{2}{|c|}{ DREDGE ROPE. } \\
\hline & & & Down. & UP. \\
\hline
\end{tabular}

$20.00 / 1.204 / 5$

200

300

400

500

600

700

800

900

1000

IIOO

I200

1300

1400

1500

1600

1700

1800

rgoo

2000

2100

2200

2300

2400 
No.

\section{Date}

Machine. Reel.
Turns
Cor. +
Depth

Shot or lead

\section{Bottom}

Bottom temperature

No. of thormometer

Cor.

Corrected temperature

$\mathscr{A i}$

Surface

Drift

Trawl or dredge

SOUNDING WIRE. DOWN. UP. TURNS. DOWN. 
noshot 